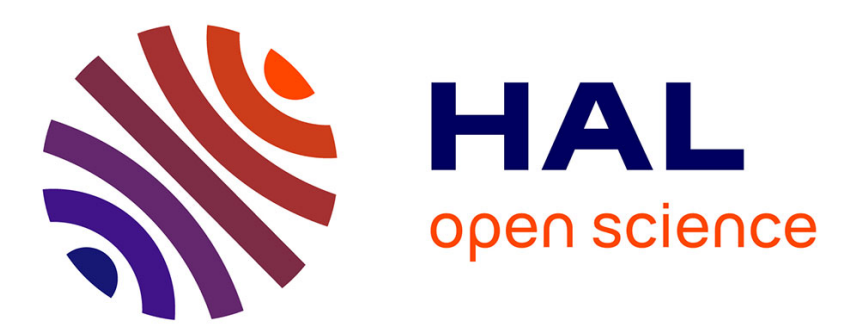

\title{
A 3D Bayesian Computed Tomography Reconstruction Algorithm with Gauss-Markov-Potts Prior Model and its Application to Real Data
}

Camille Chapdelaine, Ali Mohammad-Djafari, Nicolas Gac, Estelle Parra-Denis

\section{To cite this version:}

Camille Chapdelaine, Ali Mohammad-Djafari, Nicolas Gac, Estelle Parra-Denis. A 3D Bayesian Computed Tomography Reconstruction Algorithm with Gauss-Markov-Potts Prior Model and its Application to Real Data. Fundamenta Informaticae, 2017, 155 (4), pp.373-405. 10.3233/FI-2017-1591. hal-01569332

\section{HAL Id: hal-01569332 \\ https://hal.science/hal-01569332}

Submitted on 5 Oct 2017

HAL is a multi-disciplinary open access archive for the deposit and dissemination of scientific research documents, whether they are published or not. The documents may come from teaching and research institutions in France or abroad, or from public or private research centers.
L'archive ouverte pluridisciplinaire HAL, est destinée au dépôt et à la diffusion de documents scientifiques de niveau recherche, publiés ou non, émanant des établissements d'enseignement et de recherche français ou étrangers, des laboratoires publics ou privés. 


\title{
A 3D Bayesian Computed Tomography Reconstruction Algorithm with Gauss-Markov-Potts Prior Model and its Application to Real Data
}

\author{
Camille Chapdelaine * \\ Laboratoire des signaux et systèmes, CNRS, CentraleSupélec-Université Paris-Saclay
}

SAFRAN SA, Safran Tech, Pôle Technologie du Signal et de l'Information

Ali Mohammad-Djafari *

Laboratoire des signaux et systèmes, CNRS, CentraleSupélec-Université Paris-Saclay

Nicolas Gac *

Laboratoire des signaux et systèmes, CNRS, CentraleSupélec-Université Paris-Saclay

Estelle Parra *

SAFRAN SA, Safran Tech, Pôle Technologie du Signal et de l'Information

\begin{abstract}
Iterative reconstruction methods in Computed Tomography (CT) are known to provide better image quality than analytical methods but they are not still applied in many fields because of their computational cost. In the last years, Graphical Processor Units (GPU) have emerged as powerful devices in order to parallelize calculations, but the efficiency of their use is conditionned on applying algorithms that can be massively parallelizable. Moreover, in non-destructive testing (NDT) applications, a segmentation of the reconstructed volume is often needed in order to have an accurate diagnosis on the material health, but performing a segmentation after the reconstruction introduces uncertainties in the diagnosis from both the reconstruction and the segmentation algorithms. In this paper, we propose an iterative reconstruction method for 3D CT that performs a joint reconstruction and segmentation of the controlled object in NDT for industrial applications. The method is based on a 3D Gauss-Markov-Potts prior model in Bayesian framework, which has shown its effective use in many image restoration and super-resolution problems. First, we briefly describe this model, before deriving the expression of the joint posterior distribution of all the unknowns. Next, an effective maximization of this distribution is presented. We use a ray-driven projector and a voxel-driven backprojector implemented on GPU. The algorithm is developed so it can be massively parallelized.
\end{abstract}

Address for correspondence: C. Chapdelaine, Laboratoire des signaux et systèmes, CNRS, CentraleSupélec-Université ParisSaclay, Gif-sur-Yvette, France

${ }^{*}$ The authors are grateful to Lionel Gay and Nicolas Cochennec for having provided the real IQI phantom used to test the method. They would also like to thank Thomas Boulay for his contribution to the implementation of the projector and the backprojector on GPU. 
Finally, we present our results on simulated and real phantoms. In addition, we investigate further reconstruction quality indicators in order to compare our results with other methods.

Keywords: 3D Computed Tomography, Bayesian, Gauss-Markov-Potts, iterative CT reconstruction, X-ray

\section{Introduction}

Computed tomography $(\mathrm{CT})$ is a powerful imaging technique to see the interior of a three dimensional object and has a wide field of applications. In particular, in medicine and industry, X-rays are used to pass through the object to image, which makes their intensity decrease depending on the density they encounter in the interior. Thanks to the measurement of this intensity at the output of the acquisition process, the interior of the object is retrieved by applying what is called a reconstruction algorithm.

Many reconstruction algorithms have been developed for the last decades and can be divided in two categories. The first are analytical reconstruction methods and are based on the use of Radon transform [1, 2, 3]. In 1984, Feldkamp, Davis and Kress proposed a filtered back-projection (FBP) algorithm [4] for cone-beam CT. This algorithm is now known as FDK algorithm and is the most widely used in industry for non-destructive testing. More recently, Katsevich proposed an exact inversion algorithm for spiral cone-beam CT [5, 6], detailed in [7]. Other CT analytical algorithms use Fourier slice theorem [8] to achieve reconstruction but have to deal with a tricky interpolation from circular to cartesian gridding in Fourier space [3]. To overcome this difficulty, several convolutions of Fourier transform of the projections by a filter have been studied [9].

However, all these analytical reconstruction methods suffer from artifacts due to approximations and give poor results with limited-angle projections. From this standpoint, algebraic reconstruction techniques (ART) have been developed since the first one by Gordon, Bender and Herman [10]. These methods consider a linearized discretized model

$$
\boldsymbol{g}=\boldsymbol{H} \boldsymbol{f}+\boldsymbol{\epsilon}
$$

where $f$ is the object to retrieve. This is typically an ill-posed problem [11]. First ART like SIRT [12] or SART [13] focus on minimizing the distance between the real and estimated projections $\| \boldsymbol{g}-$ $\boldsymbol{H} \boldsymbol{f} \|^{2}$, which is a data-matching criterion. However, this does not give entirely satisfactory results, because it does not take into account any information a priori [14]. These priors can be enforced by adding a regularization term to data-matching term in the criterion to minimize. Many choices are possible for this regularizarion term, depending on the priors. For instance, some methods favour solutions with minimum $\mathcal{L}_{2}$-norm, like in [15]. Another famous prior is to enforce solutions which can be clearly divided in quite homogeneous regions. DART algorithm [16] precisely favours this type of solution by focusing on the estimation of the contours. An improvement of DART has been proposed in [17]. However, DART can be only applied for objects with finite sets of gray level. Otherwise, total variation (TV) regularization is widely used in imaging and have been successfully applied to CT in [18], by applying Chambolle and Pock algorithm [19]. Other functionals exist to enforce compact regions, like piecewise-constant Mumford-Shah functional [20]. Another noteworthy model is Potts model [21], of which the greatest interest is to perform joint reconstruction and segmentation of the object to reconstruct.

Among algebraic reconstruction techniques, we may emphasize Bayesian methods, which make the user able to take into account the errors and to estimate the uncertainty on each pixel (2D) or voxel (3D). This is a great advantage compared to non-Bayesian algebraic methods. Moreover, Bayesian framework gives a high understanding of the parameters, making their tuning easier, while it is rather tedious with non-Bayesian ART. In Bayesian framework, Gauss-Markov-Potts (GMP) model has been applied successfully in microwave imaging [22, 23] and image restoration [24, 25]. It enables to reconstruct an image or an object with finite number of materials, mapped in several compact regions. For each material, pixels (in 2D) or voxels (in 3D) of this material may vary around a mean value, but 
these variations can be controlled thanks to the hyperparameters tuning. This quite appealing simple idea has led us to implement a 3D Gauss-Markov-Potts model for CT for non-destructive testing (NDT) applications, typically in industry, based on the works done in [25]. In order to comply with industrial needs which require to obtain a high reconstruction speed, in this paper, we propose a fast and massively parallelizable algorithm. Because we know all the priors, for such models as GMP, a Gibbs sampler has been often used to perform Expectation A Posteriori (EAP) or Maximization A Posteriori (MAP) [26, 24]. However, Gibbs sampling requires more iterations as the dimension grows, and one iteration for GMP model would require to sample a Potts model, which would require thousands of iterations too. Hence, because it would require thousands of iterations which would be much longer as the dimension grows, and because industrial applications deal with very huge size volume, we show a Gibbs sampler would not be affordable in order to match industrial needs. Variational Bayesian Approach (VBA) [25] would require much more memory to keep each parameter of the approaching distribution of each unknown. All these considerations have led us to perform a joint maximization a posteriori (JMAP) for 3D Gauss-Markov-Potts model, which make able to jointly retrieve :

- a high quality estimation of the object to reconstruct

- a segmentation of the reconstructed object in compact and well-distinguishable regions

- an estimation of the means and the variances for each of these regions

In our knowledge, this has never been done before for general linear inverse problems such as CT. Moreover, Gauss-Markov-Potts prior model has been used very often in segmentation or image restoration with small size point spread function (PSF), but never in 3D for general inverse problems with real industrial number of voxels. In addition, the proposed method has the great advantage to perform the reconstruction and a segmentation of this object at the same time. Using other reconstruction methods, the segmentation has to be done after the reconstruction, which leads to cumulate two different sources of uncertainties on the classification of the voxels, i.e. the reconstruction algorithm and the segmentation algorithm, and can be a drawback for the control.

Moreover, we have noticed many papers presenting new reconstruction algorithms only qualitatively compare the gain of their methods with respect to other classical methods. Here, we take advantage of the fact that our method retrieves both a reconstruction and a segmentation to introduce quantitative reconstruction quality indicators, when comparing our results with FDK and TV methods.

The rest of the paper is organized as follows. First, we briefly describe the main prior model given in [25]. Then, the expression of the joint posterior distribution of all the unknowns is obtained. Then, we propose a joint maximization a posteriori (JMAP) algorithm to estimate the unknowns. We justify our choice of the algorithm by the huge dimension of the data. Next, we present several reconstruction quality indicators before showing our results on simulated and real phantoms.

\section{Proposed models and method}

\subsection{Forward model}

As we said, we are concerned with NDT applications of CT. Figure 1 shows the basics of the 3D reconstruction in CT, with a cone-beam acquisition process. X-rays are sended from a source and are projected on an array of detectors. Before reaching the detectors, X-rays go through an object (some industrial part) of which we want to control the interior. In order to obtain several perspectives, the object is rotated around $z$-axis, with an angle $\phi$, as shown in figure 1. By passing through the object, the intensity of X-rays decreases depending on the density of the material which compose the object, according to Beer-Lambert's law. Measurements of this intensity by the detectors at the ouput enable the tomograph to estimate the interior of the object thanks to some reconstruction algorithm (for instance, FDK algorithm [4], which is the most popular in industry). Thanks to such a process, the absence of defects in the industrial part can be controlled. 


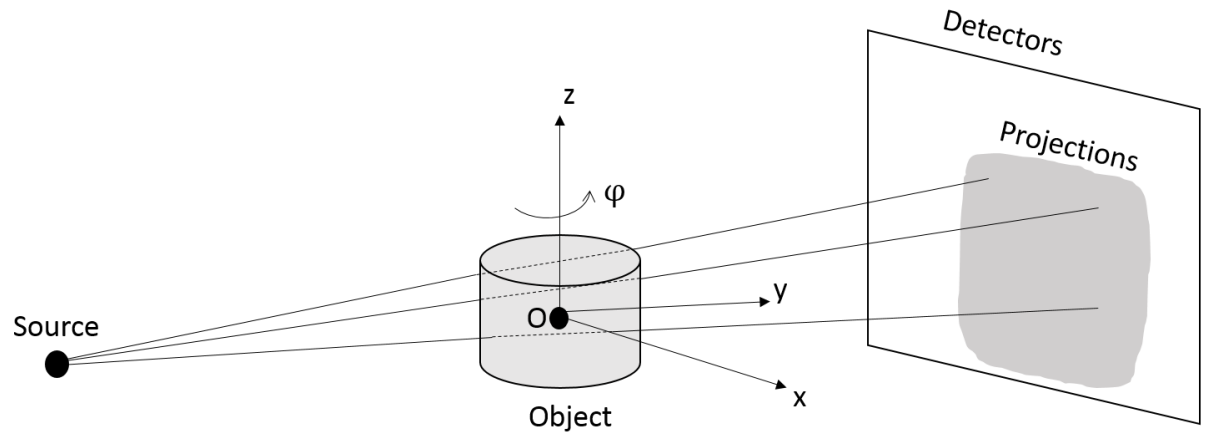

Figure 1: 3D Computed Tomography (CT) for NDT applications

We denote by $\boldsymbol{g}$ the vector of the $M$ line projections, and by $\boldsymbol{f}$ the vectorized density in the object space to reconstruct. The object is divided in $N$ voxels to be estimated in order to perform the reconstruction. By discretization of Radon transform [2,3] and accounting for the errors, we have the following model for the projections :

$$
\boldsymbol{g}=\boldsymbol{H} \boldsymbol{f}+\boldsymbol{\epsilon}
$$

where $H_{i j}$ is the length of voxel $j$ crossed by X-ray $i$. The errors $\epsilon$ are modeled as Gaussian

$$
p\left(\epsilon_{i} \mid v_{\epsilon_{i}}\right)=\mathcal{N}\left(\epsilon_{i} \mid 0, v_{\epsilon_{i}}\right), \forall i
$$

which gives the likelihood

$$
p\left(\boldsymbol{g} \mid \boldsymbol{f}, \boldsymbol{v}_{\epsilon}\right)=\mathcal{N}\left(\boldsymbol{g} \mid \boldsymbol{H} \boldsymbol{f}, \boldsymbol{V}_{\epsilon}\right)
$$

where $\boldsymbol{V}_{\epsilon}=\operatorname{diag}\left[\boldsymbol{v}_{\epsilon}\right], \boldsymbol{v}_{\epsilon}=\left(v_{\epsilon_{1}}, \ldots, v_{\epsilon_{M}}\right)$. For all measure $i$, the variance $v_{\epsilon_{i}}$ of the uncertainty is modeled as following an Inverse Gamma distribution :

$$
p\left(v_{\epsilon_{i}} \mid \alpha_{\epsilon_{0}}, \beta_{\epsilon_{0}}\right)=\mathcal{I} \mathcal{G}\left(v_{\epsilon_{i}} \mid \alpha_{\epsilon_{0}}, \beta_{\epsilon_{0}}\right) \propto v_{\epsilon_{i}}^{-\alpha_{\epsilon_{0}}-1} \exp \left[-\frac{\beta_{\epsilon_{0}}}{v_{\epsilon_{i}}}\right], \forall i
$$

where $\alpha_{\epsilon_{0}}$ and $\beta_{\epsilon_{0}}$ are fixed hyperparameters. With these assignments, we see that in fact, we are assuming $\epsilon_{i}$ follows a generalized Student-t distribution [27] :

$$
\mathcal{S} t\left(\epsilon_{i} \mid \alpha_{\epsilon_{0}}, \beta_{\epsilon_{0}}\right)=\int_{0}^{+\infty} \mathcal{N}\left(\epsilon_{i} \mid 0, v_{\epsilon_{i}}\right) \mathcal{I} \mathcal{G}\left(v_{\epsilon_{i}} \mid \alpha_{\epsilon_{0}}, \beta_{\epsilon_{0}}\right) d v_{\epsilon_{i}}
$$

\subsection{Gauss-Markov-Potts model}

In NDT application, industrial parts are composed of several materials, each material filling one or several compact regions. In order to take into account this prior information, we need to introduce common characteristic for the voxels which represent the same material (we assume that one voxel represents only one material, i.e. the resolution is sufficiently high). To do so, we use a GaussMarkov-Potts model $[24,25,23]$. This model first consists in labeling each voxel by the material it represents : that leads to introduce a hidden field $z$ so that $z_{j}=k$ if the voxel $j$ represents material $k$. In NDT applications, the number of materials in a volume to control is known : we denote it by $K$.

In order to enforce a certain homogeneity, the gray values of the voxels of a same material $k$ are distributed around a mean value $m_{k}$, with variance $v_{k}$

$$
p\left(f_{j} \mid z_{j}=k, m_{k}, v_{k}\right)=\mathcal{N}\left(f_{j} \mid m_{k}, v_{k}\right) \text { if } z_{j}=k .
$$




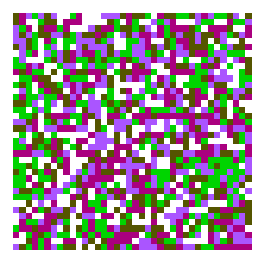

$\gamma_{0}=0.5$

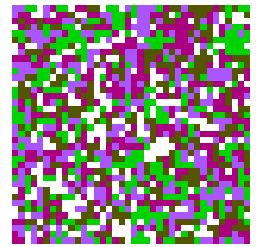

$\gamma_{0}=0.7$

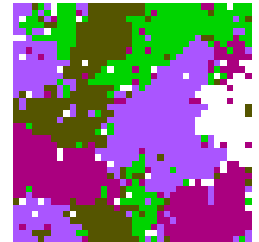

$\gamma_{0}=0.8$

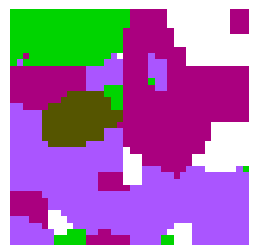

$\gamma_{0}=1.6$

Figure 2: Potts field for different values of $\gamma_{0}$

It gives the following prior for $\boldsymbol{f}$, depending on the labels $\boldsymbol{z}$, the means of the classes $\boldsymbol{m}$ and the variances of the classes $v$ :

$$
p(\boldsymbol{f} \mid \boldsymbol{z}, \boldsymbol{m}, \boldsymbol{v}) \propto \operatorname{det}\left(\boldsymbol{V}_{\boldsymbol{z}}\right)^{-1 / 2} \exp \left[-\frac{1}{2}\left\|\boldsymbol{f}-\boldsymbol{m}_{\boldsymbol{z}}\right\|_{\boldsymbol{V}_{\boldsymbol{z}}}^{2}\right]
$$

where $\boldsymbol{V}_{\boldsymbol{z}}=\operatorname{diag}\left[\boldsymbol{v}_{\boldsymbol{z}}\right]$, with $\boldsymbol{m}_{\boldsymbol{z}}$ and $\boldsymbol{v}_{\boldsymbol{z}}$ the vectors of size $N$ which correspond to $\boldsymbol{m}$ and $\boldsymbol{v}$ (of size $K$ ) distributed over the voxels according to the segmentation $z: \forall j$ and $\forall k, m_{z_{j}}=m_{k}$ and $v_{z_{j}}=v_{k}$ if $z_{j}=k$. Concerning the prior on the means and variances of the classes, we choose to model the means as gaussian

$$
p\left(m_{k} \mid m_{0}, v_{0}\right)=\mathcal{N}\left(m_{k} \mid m_{0}, v_{0}\right)
$$

and the variances as following an Inverse Gamma distribution :

$$
p\left(v_{k} \mid \alpha_{0}, \beta_{0}\right)=\mathcal{I} \mathcal{G}\left(v_{k} \mid \alpha_{0}, \beta_{0}\right)
$$

where $m_{0}, v_{0}, \alpha_{0}$ and $\beta_{0}$ are fixed parameters, depending on the object to control.

The next step is to enforce compactness, using a Markov-Potts model for hidden field $z$ :

$$
p(\boldsymbol{z}) \propto \exp \left[\sum_{j} \Phi\left(z_{j}\right)+\gamma_{0} \sum_{i \in \mathcal{V}(j)} \delta\left(z_{j}-z_{i}\right)\right]
$$

where $\mathcal{V}(j)$ is the neighbourhood of voxel $j$. The parameter $\gamma_{0}$ is called the granularity coefficient, because it has a huge influence on the granularity of the field $z$. In fact, there exists a critical value $\gamma_{c}$ for Potts coefficient $\gamma_{0}$ so that the regions are compact if $\gamma_{0} \geq \gamma_{c}$ [28, 29]. This critical value $\gamma_{c}$ can be computed for Ising field ( $K=2$ classes) in the 2D-case [30]. To illustrate this influence, figure 2 shows the influence of $\gamma_{0}$ the middle slices of generated $64 \times 64 \times 64$ Potts fields with $K=5$ classes, for different values of $\gamma_{0}$. As we see, the granularity of the field sharply changes when we increase $\gamma_{0}$ from 0.7 to 0.8 .

Concerning the energy of singleton cliques,

$$
\Phi\left(z_{j}\right)=\sum_{k=1}^{K} \alpha_{k} \delta\left(z_{j}-k\right), \forall j
$$

where $\boldsymbol{\alpha}$ is related to the proportions of each class in the hidden field $z$, which leads to the constraint, given by [25]:

$$
\sum_{k=1}^{K} \exp \left[\alpha_{k}\right]=1
$$

So, the prior for hidden Markov-Potts field (HMPF) $z$ reads

$$
p\left(\boldsymbol{z} \mid \boldsymbol{\alpha}, \gamma_{0}\right) \propto \exp \left[\sum_{j}\left(\sum_{k=1}^{K} \alpha_{k} \delta\left(z_{j}-k\right)+\gamma_{0} \sum_{i \in \mathcal{V}(j)} \delta\left(z_{j}-z_{i}\right)\right)\right]
$$




\subsection{Bayesian methods for estimation}

Given the prior model we have detailed in sections 2.1 and 2.2, many Bayesian methods exist in order to retrieve an estimation of the unknowns $f, z, \boldsymbol{v}_{\epsilon}, \boldsymbol{m}$ and $\boldsymbol{v}$, using the joint posterior distribution of the unknowns

$$
p\left(\boldsymbol{f}, \boldsymbol{z}, \boldsymbol{v}_{\epsilon}, \boldsymbol{m}, \boldsymbol{v} \mid \boldsymbol{g} ; \mathcal{M}\right)
$$

given the data $g$ and the model $\mathcal{M}$. In our case, this posterior distribution is not available. As it can be found in [31], we shall consider numerical or analytical approximations.

Analytical approximations, such as Variational Bayesian Approach (VBA), computes an approximation of the posterior distribution, which is maximized when a chosen divergence, such as KullbackLeibler divergence, does not change significantly. A completely factored distribution is often used to approximate the true posterior distribution but can lead to too gross approximations [32], since it overlooks the correlation between each variables by the mean-field approximation (MFA) [33, 34]. Moreover, VBA requires much memory in order to keep each parameter of each approaching distribution in the fully factored model.

On the opposite, numerical methods try to deal with the true posterior distribution, by Monte Carlo Markov Chain (MCMC) methods, or Joint Maximization A Posteriori (JMAP). JMAP try to maximize the posterior distribution with respect to each unknown iteratively, as it is done in equation (25) in section 3. MCMC methods aim at computing MMSE (Minimum Mean Square Error) or MAP (Maximum A Posteriori) estimators by generating samples of the posterior distribution. When all the priors are known, this often consists in a Gibbs sampler. These methods are more reliable than JMAP but have to pick many samples of the conditional distributions of each unknown given the others. In addition, they require more samples as the dimension grows. In our case, the very huge size of the controlled volume result in thousands of iterations of which each one would be very long. In the sequel, in section 3, we emphasize how a Gibbs sampling would be very costly in terms of computation time compared with JMAP. This huge cost makes MCMC methods unaffordable for our industrial NDT application and leads us to consider JMAP in order to estimate all the unknowns by maximizing their joint posterior distribution.

\section{Joint maximization of the posterior distribution}

\subsection{Algorithm for the estimation of the unknowns}

Our model is summarized in figure 3. By applying Bayes's rule, the posterior distribution of all the unknowns $\boldsymbol{f}, \boldsymbol{z}, \boldsymbol{v}_{\epsilon}, \boldsymbol{m}$ and $\boldsymbol{v}$ reads

$$
p\left(\boldsymbol{f}, \boldsymbol{z}, \boldsymbol{v}_{\epsilon}, \boldsymbol{m}, \boldsymbol{v} \mid \boldsymbol{g} ; \mathcal{M}\right)=\frac{p\left(\boldsymbol{g}, \boldsymbol{f}, \boldsymbol{z}, \boldsymbol{v}_{\epsilon}, \boldsymbol{m}, \boldsymbol{v} \mid \mathcal{M}\right)}{p(\boldsymbol{g} \mid \mathcal{M})} .
$$

Our prior model appears in

$$
\begin{aligned}
p\left(\boldsymbol{g}, \boldsymbol{f}, \boldsymbol{z}, \boldsymbol{v}_{\epsilon}, \boldsymbol{m}, \boldsymbol{v} \mid \mathcal{M}\right)= & p\left(\boldsymbol{g} \mid \boldsymbol{f}, \boldsymbol{v}_{\epsilon}\right) p(\boldsymbol{f} \mid \boldsymbol{z}, \boldsymbol{m}, \boldsymbol{v}) \\
& p\left(\boldsymbol{v}_{\epsilon} \mid \alpha_{\epsilon_{0}}, \beta_{\epsilon_{0}}\right) p\left(\boldsymbol{z} \mid \boldsymbol{\alpha} ; \gamma_{0}\right) \\
& p\left(\boldsymbol{m} \mid m_{0}, v_{0}\right) p\left(\boldsymbol{v} \mid \alpha_{0}, \beta_{0}\right)
\end{aligned}
$$

with

$$
\begin{gathered}
p\left(\boldsymbol{g} \mid \boldsymbol{f}, \boldsymbol{v}_{\epsilon}\right) \propto \operatorname{det}\left(\boldsymbol{V}_{\epsilon}\right)^{-1 / 2} \exp \left[-\frac{1}{2}\|\boldsymbol{g}-\boldsymbol{H} \boldsymbol{f}\|_{\boldsymbol{V}_{\epsilon}}^{2}\right] \\
p(\boldsymbol{f} \mid \boldsymbol{z}, \boldsymbol{m}, \boldsymbol{v}) \propto \operatorname{det}\left(\boldsymbol{V}_{\boldsymbol{z}}\right)^{-1 / 2} \exp \left[-\frac{1}{2}\left\|\boldsymbol{f}-\boldsymbol{m}_{\boldsymbol{z}}\right\|_{\boldsymbol{V}_{\boldsymbol{z}}}^{2}\right] \\
p\left(\boldsymbol{v}_{\epsilon} \mid \alpha_{\epsilon_{0}}, \beta_{\epsilon_{0}}\right) \propto \exp \left[-\sum_{i}\left(\left(\alpha_{\epsilon_{0}}+1\right) \ln \left(v_{\epsilon_{i}}\right)+\frac{\beta_{\epsilon_{0}}}{v_{\epsilon_{i}}}\right)\right]
\end{gathered}
$$




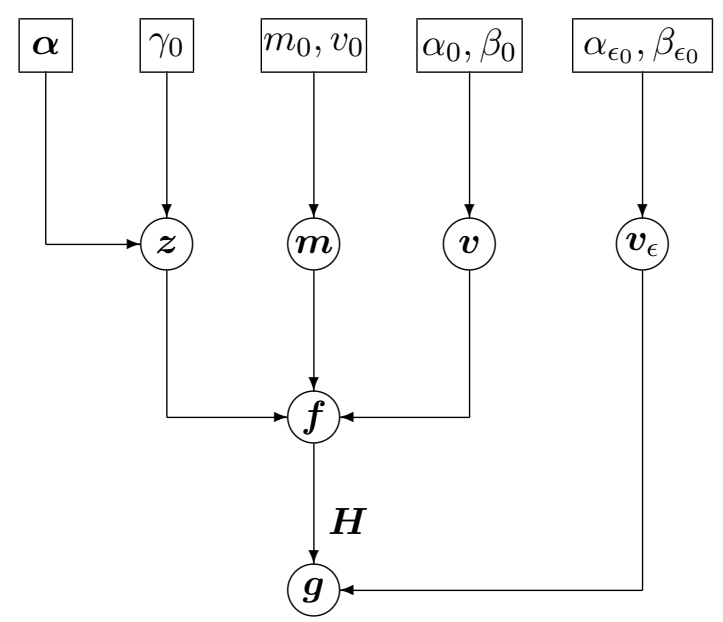

Figure 3: Hierarchical Gauss-Markov-Potts prior model

$$
\begin{gathered}
p\left(\boldsymbol{z} \mid \boldsymbol{\alpha}, \gamma_{0}\right) \propto \exp \left[\sum_{j}\left(\sum_{k=1}^{K} \alpha_{k} \delta\left(z_{j}-k\right)+\gamma_{0} \sum_{i \in \mathcal{V}(j)} \delta\left(z_{j}-z_{i}\right)\right)\right] \\
p\left(\boldsymbol{m} \mid m_{0}, v_{0}\right) \propto v_{0}^{-\frac{K}{2}} \exp \left[-\frac{1}{2 v_{0}} \sum_{k=1}^{K}\left(m_{k}-m_{0}\right)^{2}\right], \\
p\left(\boldsymbol{v} \mid \alpha_{0}, \beta_{0}\right) \propto \exp \left[-\sum_{k=1}^{K}\left(\left(\alpha_{0}+1\right) \ln v_{k}+\frac{\beta_{0}}{v_{k}}\right)\right] .
\end{gathered}
$$

To estimate the unknowns $\boldsymbol{f}, \boldsymbol{z}, \boldsymbol{v}_{\epsilon}, \boldsymbol{m}$ and $\boldsymbol{v}$, which such a prior model, it is common to use a Gibbs sampler, which consists in, at the $t^{t h}$ global iteration :

$$
\left\{\begin{array}{l}
\boldsymbol{f}^{(t)} \sim p\left(\boldsymbol{f} \mid \boldsymbol{z}^{(t-1)}, \boldsymbol{v}_{\epsilon}^{(t-1)}, \boldsymbol{m}^{(t-1)}, \boldsymbol{v}^{(t-1)} ; \boldsymbol{g}, \mathcal{M}\right) \\
\boldsymbol{z}^{(t)} \sim p\left(\boldsymbol{z} \mid \boldsymbol{f}^{(t)}, \boldsymbol{v}_{\epsilon}^{(t-1)}, \boldsymbol{m}^{(t-1)}, \boldsymbol{v}^{(t-1)} ; \boldsymbol{g}, \mathcal{M}\right) \\
\boldsymbol{v}_{\epsilon}^{(t)} \sim p\left(\boldsymbol{v}_{\epsilon} \mid \boldsymbol{f}^{(t)}, \boldsymbol{z}^{(t)}, \boldsymbol{m}^{(t-1)}, \boldsymbol{v}^{(t-1)} ; \boldsymbol{g}, \mathcal{M}\right) \\
\boldsymbol{m}^{(t)} \sim p\left(\boldsymbol{m} \mid \boldsymbol{f}^{(t)}, \boldsymbol{z}^{(t)}, \boldsymbol{v}_{\epsilon}^{(t)}, \boldsymbol{v}^{(t-1)} ; \boldsymbol{g}, \mathcal{M}\right) \\
\boldsymbol{v}^{(t)} \sim p\left(\boldsymbol{v} \mid \boldsymbol{f}^{(t)}, \boldsymbol{z}^{(t)}, \boldsymbol{v}_{\epsilon}^{(t)}, \boldsymbol{m}^{(t)} ; \boldsymbol{g}, \mathcal{M}\right)
\end{array}\right.
$$

This has been done, for instance, in microwave imaging [22], diffraction imaging [26] and ultrasound imaging [35] : after a heating time, the algorithm (23) generates samples that can be used to compute MAP or MMSE estimators. The problem of Gibbs sampling is that it requires more samples as the dimension grows. In our application in 3D, the controlled volumes have very huge sizes : in section 5.3, we show results on volumes with $256^{3}$ and $512 \times 512 \times 256$ voxels, but we are eager to use our algorithm on volumes with $1024^{3}$ or $2048^{3}$ voxels when it will be fully parallelized. So, Gibbs sampling would require thousands of cycles (i.e. global iterations) to converge.

Moreover, only one cycle would take very long time too. Indeed, sampling $f$ with respect to its posterior distribution given the other unknpwns leads to sampling a Gaussian field (because of the conjugate priors) in very huge dimension. To do that in an efficient way, a perturbation-optimization method $[36,37]$ has been proposed to compute one sample $f$ by solving a linear system, in which the data and the prior mean have been perturbed. The problem is this technique makes the quality of the sample dependent on the number of iterations of the gradient descent to solve the system. To take into account this approximation, an accept-reject strategy is developed in [38], but the use of this strategy to sample $f$ would increase the computation time. In addition, sampling $z$ with respect 
to $p\left(\boldsymbol{z} \mid \boldsymbol{f}, \boldsymbol{v}_{\epsilon}, \boldsymbol{m}, \boldsymbol{v} ; \boldsymbol{g}, \mathcal{M}\right)$ would require thousands of iterations. For instance, in [39], by applying the method of [40] for a little volume of about $100^{3}$ voxels, the burn-in period to sample correct labels with respect to the conditional distribution given all the other unknowns represents nearly 1000 iterations. In [35], Gibbs sampling is applied for a similar Potts model in ultrasound imaging and $z$ is sampled as it is done in [26]: the computational complexity for sampling $z$ in one cycle is estimated to be of the order $\mathcal{O}(K N)$. In our application, $N$ is very huge $\left(256^{3}, 512^{3}, 1024^{3}, 2048^{3}, \ldots\right)$. Thereby, in our case, this step in one cycle of Gibbs sampling cannot be achieved in reasonable time.

As we have seen, one cycle of a Gibbs sampling would take very much time. Given that we would need thousands of these cycles in order to obtain correct samples of the joint posterior distribution of the unknowns $\boldsymbol{f}, \boldsymbol{z}, \boldsymbol{v}_{\epsilon}, \boldsymbol{m}$ and $\boldsymbol{v}$, a Gibbs sampling would be infinitely long. Hence, a Gibbs sampling is not affordable in our industrial application, which needs fast reconstruction algorithms. That is why we chose to perform a joint maximization of the posterior distribution of all the unknowns :

$$
\left(\hat{\boldsymbol{f}}, \hat{\boldsymbol{z}}, \hat{\boldsymbol{v}}_{\epsilon}, \hat{\boldsymbol{m}}, \hat{\boldsymbol{v}}\right)=\underset{\left(\boldsymbol{f}, \boldsymbol{z}, \boldsymbol{v}_{\epsilon}, \boldsymbol{m}, \boldsymbol{v}\right)}{\arg \max }\left\{p\left(\boldsymbol{f}, \boldsymbol{z}, \boldsymbol{v}_{\epsilon}, \boldsymbol{m}, \boldsymbol{v} \mid \boldsymbol{g}, \mathcal{M}\right)\right\}
$$

by doing the approximate algorithm :

$$
\left\{\begin{array}{l}
\boldsymbol{f}^{(t)} \leftarrow \arg \max _{\boldsymbol{f}}\left\{p\left(\boldsymbol{f} \mid \boldsymbol{z}^{(t-1)}, \boldsymbol{v}_{\epsilon}^{(t-1)}, \boldsymbol{m}^{(t-1)}, \boldsymbol{v}^{(t-1)} ; \boldsymbol{g}, \mathcal{M}\right)\right\} \\
\boldsymbol{z}^{(t)} \leftarrow \arg \max _{\boldsymbol{z}}\left\{p\left(\boldsymbol{z} \mid \boldsymbol{f}^{(t)}, \boldsymbol{v}_{\epsilon}^{(t-1)}, \boldsymbol{m}^{(t-1)}, \boldsymbol{v}^{(t-1)} ; \boldsymbol{g}, \mathcal{M}\right)\right\} \\
\boldsymbol{v}_{\epsilon}^{(t)} \leftarrow \arg \max _{\boldsymbol{v}_{\epsilon}}\left\{p\left(\boldsymbol{v}_{\epsilon} \mid \boldsymbol{f}^{(t)}, \boldsymbol{z}^{(t)}, \boldsymbol{m}^{(t-1)}, \boldsymbol{v}^{(t-1)} ; \boldsymbol{g}, \mathcal{M}\right)\right\} \\
\boldsymbol{m}^{(t)} \leftarrow \arg \max _{\boldsymbol{m}}\left\{p\left(\boldsymbol{m} \mid \boldsymbol{f}^{(t)}, \boldsymbol{z}^{(t)}, \boldsymbol{v}_{\epsilon}^{(t)}, \boldsymbol{v}^{(t-1)} ; \boldsymbol{g}, \mathcal{M}\right)\right\} \\
\boldsymbol{v}^{(t)} \leftarrow \arg \max _{\boldsymbol{v}}\left\{p\left(\boldsymbol{v} \mid \boldsymbol{f}^{(t)}, \boldsymbol{z}^{(t)}, \boldsymbol{v}_{\epsilon}^{(t)}, \boldsymbol{m}^{(t)} ; \boldsymbol{g}, \mathcal{M}\right)\right\}
\end{array}\right.
$$

This joint maximization a posteriori (JMAP) requires less computation time than a Gibbs sampler. The other side of the coin is the algorithm is dependent on the initialization, which has to be sufficiently good. This matter is discussed in section 5.2. In the rest of this section, we describe each step of this algorithm. After having defined reconstruction quality indicators using the estimated volume $f$ and the estimated labels $z$, we present results obtained on simulated and real data in section 5.3.

\subsection{Estimation of the object $f$}

The posterior distribution for the object $f$

$$
p\left(\boldsymbol{f} \mid \boldsymbol{z}, \boldsymbol{v}_{\epsilon}, \boldsymbol{m}, \boldsymbol{v} ; \boldsymbol{g}, \mathcal{M}\right) \propto p\left(\boldsymbol{g} \mid \boldsymbol{f}, \boldsymbol{v}_{\epsilon}\right) p(\boldsymbol{f} \mid \boldsymbol{z}, \boldsymbol{m}, \boldsymbol{v})
$$

gives the criterion to minimize in order to retrieve the estimation of the object, given all the other unknowns :

$$
J(\boldsymbol{f})=\|\boldsymbol{g}-\boldsymbol{H} \boldsymbol{f}\|_{\boldsymbol{V}_{\epsilon}}^{2}+\left\|\boldsymbol{f}-\boldsymbol{m}_{\boldsymbol{z}}\right\|_{\boldsymbol{V}_{\boldsymbol{z}}}^{2}
$$

The gradient reads

$$
\nabla J(\boldsymbol{f})=2\left(\boldsymbol{H}^{T} \boldsymbol{V}_{\epsilon}^{-1} \boldsymbol{H}+\boldsymbol{V}_{\boldsymbol{z}}^{-1}\right) \boldsymbol{f}-2\left(\boldsymbol{V}_{\boldsymbol{z}}^{-1} \boldsymbol{m}_{\boldsymbol{z}}+\boldsymbol{H}^{T} \boldsymbol{V}_{\epsilon}^{-1} \boldsymbol{g}\right) .
$$

It gives the analytical expression of the optimal object which minimizes the criterion :

$$
\boldsymbol{f}^{*}=\left(\boldsymbol{H}^{T} \boldsymbol{V}_{\epsilon}^{-1} \boldsymbol{H}+\boldsymbol{V}_{\boldsymbol{z}}^{-1}\right)^{-1}\left(\boldsymbol{H}^{T} \boldsymbol{V}_{\epsilon}^{-1} \boldsymbol{g}+\boldsymbol{V}_{\boldsymbol{z}}^{-1} \boldsymbol{m}_{\boldsymbol{z}}\right)
$$

Because of the huge dimension, this expression is not suitable for our application : that is why we decide to minimize the criterion by a simple gradient descent. We give details about this algorithm in appendix A. 


\subsection{Estimation of the hidden Potts field $z$}

As for the object, we compute the posterior distribution of the Potts field $z$ given all the other unknowns

$$
p\left(\boldsymbol{z} \mid \boldsymbol{f}, \boldsymbol{v}_{\epsilon}, \boldsymbol{m}, \boldsymbol{v} ; \boldsymbol{g}, \mathcal{M}\right) \propto p(\boldsymbol{f} \mid \boldsymbol{z}, \boldsymbol{m}, \boldsymbol{v}) p\left(\boldsymbol{z} \mid \boldsymbol{\alpha} ; \gamma_{0}\right)
$$

$p\left(\boldsymbol{z} \mid \boldsymbol{\alpha}, \gamma_{0}\right)$ is given by equation (14). The likelihood is

$$
\begin{aligned}
& \ln (p(\boldsymbol{f} \mid \boldsymbol{z}, \boldsymbol{m}, \boldsymbol{v})) \propto-\frac{1}{2} \sum_{k=1}^{K} \sum_{j \in \mathcal{R}_{k}}\left(\frac{\left(f_{j}-m_{k}\right)^{2}}{v_{k}}+\ln \left(v_{k}\right)\right) \\
& \propto-\frac{1}{2} \sum_{j} \sum_{k=1}^{K} \delta\left(z_{j}-k\right)\left(\frac{\left(f_{j}-m_{k}\right)^{2}}{v_{k}}+\ln \left(v_{k}\right)\right)
\end{aligned}
$$

So, we need to maximize Potts energy with respect to $z$ in order to estimate the segmentation

$$
J(\boldsymbol{z})=\sum_{j}\left[\sum_{k=1}^{K}\left(\alpha_{k}-\frac{\left(f_{j}-m_{k}\right)^{2}}{2 v_{k}}-\frac{1}{2} \ln \left(v_{k}\right)\right) \delta\left(z_{j}-k\right)+\gamma_{0} \sum_{i \in \mathcal{V}(j)} \delta\left(z_{j}-z_{i}\right)\right]
$$

By denoting

$$
\alpha_{j k}=\alpha_{k}-\frac{\left(f_{j}-m_{k}\right)^{2}}{2 v_{k}}-\frac{1}{2} \ln \left(v_{k}\right)
$$

we can rewrite this energy

$$
J(\boldsymbol{z})=\sum_{j}\left[\sum_{k=1}^{K} \alpha_{j k} \delta\left(z_{j}-k\right)+\gamma_{0} \sum_{i \in \mathcal{V}(j)} \delta\left(z_{j}-z_{i}\right)\right] .
$$

We now have to choose an algorithm to estimate the labels $\boldsymbol{z}$, which are markovian. Because we deal with huge size data, again, the chosen algorithm has to be fast and massively parallelizable.

The maximization of Potts energy has been dealt with in [41] using a graph-cut method. In this algorithm, the possible labels and the classes of the voxels are gathered in a same graph. Each voxel $j$ and label $k$ are linked by an edge weighted by the term $\alpha_{j k}$ and each neighboring voxels by an edge weighted by the term $\gamma_{0}$. When assigning a class to the voxels, the graph is cut to link only a voxel and its class, and neighboring voxels which have the same class. Potts energy of the segmentation corresponds to the sum of the weights of the cut. Hence, as it is done in [42], finding the optimum of Potts energy leads to finding the optimal cut, which corresponds to the graph with maximum flow. This can be done by using, for instance, the algorithm proposed by Ford and Fulkerson [43]. To move from one segmentation to another, Boykov et al. propose $\alpha \beta$-swaps and $\alpha$-expansion [41]. Unfortunately, memory is a limiting factor of maximum flow algorithms [44]. Moreover, although parallel implementations of graph-cut have been proposed (see [44] for instance), a fast implementation of maximum flow algorithms is probably not possible [45].

Another well-known segmentation algorithm is Iterated Conditional Modes (ICM) [46], which has obtained good results in the past decades [47, 48, 49]. This algorithm has been often compared with Simulated Annealing (SA) [50] which reaches global optimum but requires too much computing time [47]. The idea of ICM is to divide the voxels into two subsets, called "black" voxels and "white" voxels. These subsets are such that the neighbours of a "white" voxel are only "black" voxels, and vice versa. Figure 4, taken from [24], illustrates this subdivision. We denote by $\boldsymbol{z}_{N}$ the segmentation for "black" voxels, and by $\boldsymbol{z}_{B}$ the segmentation for "white" voxels. After having done this subdivision, it is obvious that, given the "white" voxels, "black" voxels are independent from each other, and this is the same for "white" voxels given the "black" voxels. The labellings for "white" voxels and "black" voxels are performed one after the other, as shown in figure 5. This algorithm is clearly massively parallelizable because "white" voxels (respectively "black" voxels) in figure 4 can be labelled at the same time. Its main disadvantage is its local nature, which make the initial segmentation crucial. Its highly-parallelizable nature is the main reason why we have chosen to apply ICM in order to estimate $z$ given the other unknowns, although $\alpha$-expansion and $\alpha \beta$-swap both dominate it [51]. We repeat ICM as long as Potts energy in equation (32) changes significantly. 


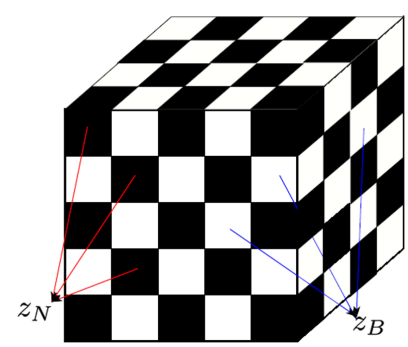

Figure 4: Subvision in white and black voxels, to perform the segmentation

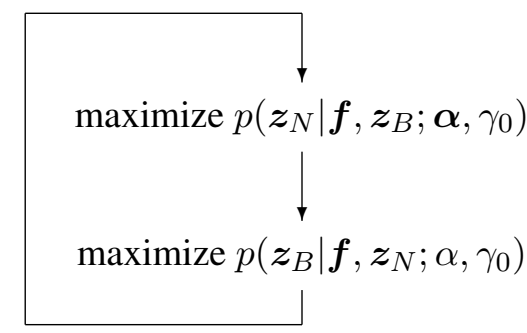

Figure 5: Segmentation algorithm for JMAP

\subsection{Estimation of the other unknowns}

Thanks to conjugate prior, the estimation of the other unknowns $\boldsymbol{v}_{\epsilon}, \boldsymbol{m}$ and $\boldsymbol{v}$ is very straightforward and we obtain the following formulas. Concerning the variances of the noise $\boldsymbol{v}_{\epsilon}$,

$$
p\left(v_{\epsilon_{i}} \mid \boldsymbol{f}, \boldsymbol{z}, \boldsymbol{m}, \boldsymbol{v} ; \boldsymbol{g}, \mathcal{M}\right)=\mathcal{I} \mathcal{G}\left(v_{\epsilon_{i}} \mid \hat{\alpha}_{\epsilon_{0_{i}}}, \hat{\beta}_{\epsilon_{0_{i}}}\right), \forall i
$$

with

$$
\left\{\begin{array}{l}
\hat{\alpha}_{\epsilon_{0_{i}}}=\alpha_{\epsilon_{0}}+\frac{1}{2} \\
\hat{\beta}_{\epsilon_{0_{i}}}=\beta_{\epsilon_{0}}+\frac{1}{2}\left(g_{i}-[\boldsymbol{H} \boldsymbol{f}]_{i}\right)^{2} \quad, \forall i
\end{array}\right.
$$

which gives the estimation $\hat{v}_{\epsilon_{i}}$ of the variance of the $i^{t h}$ measurement, by maximizing the posterior distribution $p\left(v_{\epsilon_{i}} \mid \boldsymbol{f}, \boldsymbol{z}, \boldsymbol{m}, \boldsymbol{v} ; \boldsymbol{g}, \mathcal{M}\right)$

$$
\hat{v}_{\epsilon_{i}}=\frac{\beta_{\epsilon_{0}}+\frac{1}{2}\left(g_{i}-[\boldsymbol{H} \boldsymbol{f}]_{i}\right)^{2}}{\alpha_{\epsilon_{0}}+\frac{3}{2}}, \forall i .
$$

Then, we estimate the means of the classes. We compute the expression of their posterior distribution

$$
p\left(m_{k} \mid \boldsymbol{f}, \boldsymbol{z}, \boldsymbol{v}_{\epsilon}, \boldsymbol{v} ; m_{0}, v_{0}\right)=\mathcal{N}\left(m_{k} \mid \hat{m}_{0_{k}}, \hat{v}_{0_{k}}\right),
$$

$\forall k \in\{1, \ldots, K\}$, with

$$
\left\{\begin{array}{l}
\hat{m}_{0_{k}}=\frac{\frac{1}{v_{k}} \sum_{j \in \mathcal{R}_{k}} f_{j}+\frac{m_{0}}{v_{0}}}{\frac{N_{k}}{v_{k}}+\frac{1}{v_{0}}} \\
\hat{v}_{0_{k}}=\frac{1}{\frac{N_{k}}{v_{k}}+\frac{1}{v_{0}}}
\end{array} \quad, \forall k \in\{1, \ldots, K\}\right.
$$

where $\mathcal{R}_{k}$ denotes the set of voxels which are in class $k$ and $N_{k}=\left|\mathcal{R}_{k}\right|$ the number of voxels in class $k$. So we have

$$
\hat{m}_{k}=\hat{m}_{0_{k}}=\frac{m_{0}+\frac{v_{0}}{v_{k}} \sum_{j \in \mathcal{R}_{k}} f_{j}}{1+\frac{N_{k} v_{0}}{v_{k}}}, \forall k \in\{1, \ldots, K\} .
$$


The last step is the estimation of the variances of the classes. Their posterior distribution is

$$
p\left(v_{k} \mid \boldsymbol{f}, \boldsymbol{z}, \boldsymbol{v}_{\epsilon}, \boldsymbol{m} ; \alpha_{0}, \beta_{0}\right)=\mathcal{I} \mathcal{G}\left(v_{k} \mid \hat{\alpha}_{0_{k}}, \hat{\beta}_{0_{k}}\right)
$$

with

$$
\left\{\begin{array}{l}
\hat{\alpha}_{0_{k}}=\alpha_{0}+\frac{N_{k}}{2} \\
\hat{\beta}_{0_{k}}=\beta_{0}+\frac{1}{2} \sum_{j \in \mathcal{R}_{k}}\left(f_{j}-m_{k}\right)^{2}
\end{array}, \forall k \in\{1, \ldots, K\}\right.
$$

So,

$$
\hat{v}_{k}=\frac{\beta_{0}+\frac{1}{2} \sum_{j \in \mathcal{R}_{k}}\left(f_{j}-m_{k}\right)^{2}}{\alpha_{0}+\frac{N_{k}}{2}+1}, \forall k \in\{1, \ldots, K\} .
$$

\subsection{Summary of the algorithm}

We now have all the ingredients to perform JMAP algorithm of equation (25). After having obtained a first estimation of the volume $\boldsymbol{f}^{(0)}$, the variances of the noise $\boldsymbol{v}_{\epsilon}^{(0)}$ are estimated and a segmentation of the initial volume $\boldsymbol{f}^{(0)}$ is performed, which gives $\boldsymbol{z}^{(0)}$. Using $\boldsymbol{f}^{(0)}$ and $\boldsymbol{z}^{(0)}$, the initial means $\boldsymbol{m}^{(0)}$ and variances $\boldsymbol{v}^{(0)}$ of the classes are computed. As we said, because the algorithm, which is detailed by algorithm 1, finds a local optimum, the initial volume and segmentation has to be good enough. This point is discussed in section 5.2.

\section{Indicators to evaluate the quality of the reconstruction}

In order to evaluate the performances of the reconstruction algorithm, we define some indicators for evaluating the quality of the reconstruction. To our mind, a good reconstruction quality indicator should agree with the judgement we make when just looking at the reconstruction. In our knowledge, this matter has not been far-investigated. Here, we define four indicators.

A common indicator is the relative $\mathcal{L}_{p}$-difference between the actual and the estimated projections :

$$
\Delta_{p} \boldsymbol{g}=\frac{\|\boldsymbol{g}-\boldsymbol{H} \boldsymbol{f}\|_{p}^{p}}{\|\boldsymbol{g}\|_{p}^{p}}
$$

Here, we use $p=2$. Because CT is an ill-posed problem, it is known that $\Delta_{p} \boldsymbol{g}$ is not an optimal reconstruction quality indicator.

In order to investigate this matter further, we here define three indicators which do not need reference in order to apply it in real-life applications. These indicators use the segmentation estimated by our algorithm. Since the segmentation is estimated jointly with the reconstructed object (i.e. the quality of the reconstruction depends on the quality of the reconstruction and vice versa), a good reconstruction implies a good segmentation and vice versa. The first indicator we define is a compactness indicator which computes for each voxel $j$ in class $k$ the percentage of its neighbours which are in the same class

$$
\frac{1}{N_{\mathcal{V}}} \sum_{i \in \mathcal{V}(j)} \delta\left(k-z_{i}\right)
$$

where $N_{\mathcal{V}}$ denotes the number of neighbours. Here, $N_{\mathcal{V}}=6$. Then, we compute the average of this percentage over all the voxels of class $k$ and then over all the classes. Finally, it gives the compactness indicator

$$
C_{\text {omp }}=\frac{1}{K} \sum_{k=1}^{K} \frac{1}{N_{k}} \sum_{j \in \mathcal{R}_{k}} \frac{1}{N_{\mathcal{V}}} \sum_{i \in \mathcal{V}(j)} \delta\left(k-z_{i}\right)
$$

When computing a reconstruction, we are eager to obtain regions as compact as possible. So, in our

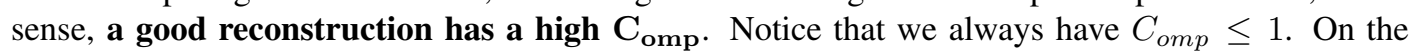




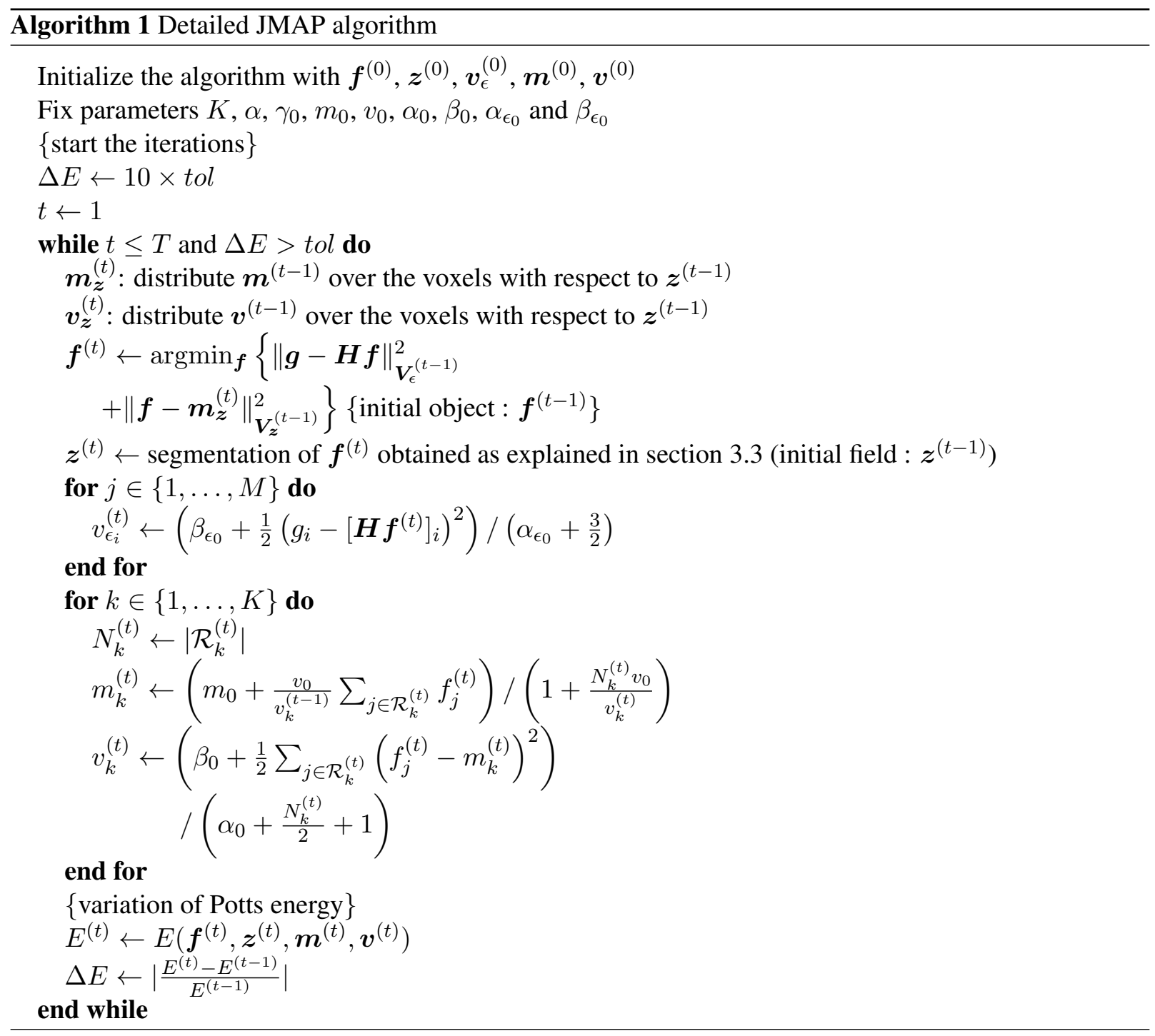

opposite, no compact classes are seen as defaults due to the reconstruction algorithm. That is why in the formula of $C_{o m p}$, an uniform average over the classes is performed, so that no compact classes strongly penalize the reconstruction.

Next, once we have compact classes, one may be interested to focus on the contours of the regions : on the contours, do we have different classes's voxels well-distinguishable ? To take into account this requirement, for one voxel $j$ in class $k$ and one of its neighbours $i$ not in the same class as it, we compute a penalization term

$$
\exp \left(-\left(f_{j}-f_{i}\right)^{2}\right)
$$

which is low if the classes of $j$ and $i$ are well-distinguishable, and high (i.e. close to 1 ) if not. We next compute the average of this term over all the neighbours of $j$ which are not in the class as $i$

$$
\left(\bar{d}_{i s t}\right)_{j}=\left\{\begin{array}{l}
\frac{\sum_{i \in \mathcal{V}(j)} \exp \left(-\left(f_{j}-f_{i}\right)^{2}\right)\left(1-\delta\left(k-z_{i}\right)\right)}{\sum_{i \in \mathcal{V}(j)}\left(1-\delta\left(k-z_{i}\right)\right)} \text { if } j \text { is on a contour }\left(z_{j}=k\right) \\
0 \text { otherwise }
\end{array}\right.
$$

Like for compactness indicator, we next perform the average over class $k$ and over the classes, and we 
define the distinguishability indicator as

$$
d_{i s t}=1-\frac{1}{K} \sum_{k=1}^{K} \frac{1}{N_{k}} \sum_{j \in \mathcal{R}_{k}}\left(\bar{d}_{i s t}\right)_{j}
$$

in order to have an indicator which is high for good reconstructions : the higher $d_{\text {ist }}$ is, the better the reconstruction is. This indicator $d_{i s t}$ favours reconstructions with well-distinguishable contours. Still, we compute an uniform average over the classes to penalize the classes which are not distinguishable from the others.

At last, we are eager to see if voxels in the same class have near gray levels. That is what we aim at when defining the homogeneity indicator

$$
h_{o m o}=\frac{1}{K} \sum_{k=1}^{K} \frac{1}{N_{k}} \sum_{j \in \mathcal{R}_{k}}\left(\hat{h}_{o m o}\right)_{j}
$$

with, if $z_{j}=k$ :

$$
\left(\hat{h}_{\text {omo }}\right)_{j}=\left\{\begin{array}{l}
\frac{\sum_{i \in \mathcal{V}(j)} \exp \left(-\left(f_{j}-f_{i}\right)^{2}\right) \delta\left(k-z_{i}\right)}{\sum_{i \in \mathcal{V}(j)} \delta\left(k-z_{i}\right)} \\
0 \text { otherwise }
\end{array}\right.
$$

Once again, $h_{\text {omo }}$ is such that the higher $\mathbf{h}_{\text {omo }}$ is, the better the reconstruction is.

In total, we have defined three new reconstruction quality indicators which exploits the segmentation estimated jointly with the volume. In section 5 , when comparing our method with others, we calculate these indicators for each reconstruction, in order to see if they agree with visual judgement.

\section{Results}

Here, we test our method on simulated and real volumes. The simulated volume is a Shepp-Logan phantom [52] in 3D, with $256^{3}$ voxels, shown in figure 8 , with its corresponding segmentation in $K=5$ classes. For this volume, 64 projections are used to do the reconstruction.

The real volume volume is a 3D IQI phantom [53], with size $512 \times 512 \times 256$ voxels. 300 uniformlydistributed projections have been acquired. Each projection is an image with $512 \times 512$ pixels, like the ones in figure 15 . The real volume is shown in figure $14:$ it has been obtained by FDK reconstruction method [4] from 2400 projections.

\subsection{Details of implementation}

Because we deal with huge dimension object, matrix $\boldsymbol{H}$ is impossible to keep in memory. Consequently, we need to implement a projection operator (operator $\boldsymbol{H}$ ) and a backprojection operator (operator $\boldsymbol{H}^{T}$ ) to perform the object estimation step in JMAP algorithm (in section 3.2). Here, speed of these two operators arises as a key point for the efficiency of the algorithm.

The core of algorithm speed up consists in implementing forward projection and backprojection on GPU hardware, using CUDA to have high computing performances [15]. Details about types of projector and backprojector and their implementation can be found in $[15,54,55,56,57,58,59]$.

As in [15], the projector we use here is ray-driven, i.e. one thread on GPU handles the ray-tracing of one ray. In one thread, the considered ray is regularly sampled in the volume in $n_{k}$ points. At each point on the ray, the value of the volume is calculated by a trilinear interpolation. Then, this computed value multiplicated by the step-size between two consecutive sample points is added to the value of the projection, according to the discretized Beer-Lambert law. A scheme of this ray-driven projector is shown in figure 6 , in 2D for the sake of readability. Concerning the backprojector, once again as in 
[15], it is voxel-driven, i.e. one thread handles one voxel. In one thread, as it is presented in figure 7 in $2 \mathrm{D}$, for each projection angle $\phi$, we trace a ray between the source and the considered voxel and we project it on the plan of detectors. The value of the obtained projection is computed by a bilinear interpolation. This value is added to the backprojected volume for the considered voxel. As we see, in our implementation, projector and backprojector are unmatched, i.e. backprojection operator is not exactly $\boldsymbol{H}^{T}$. This can be a problem to well-estimate the object [59], but we did not notice troubles due to that.

We may underline that the segmentation step is done on CPU. A good way to speed up the algorithm would be to implement this step on GPU too. This will be done in future works.

\subsection{Strategies for the initialization of the algorithm and choice of the param- eters}

The initialization is a key step when applying a local optimization algorithm. Here, JMAP algorithm needs an initial volume and an initial segmentation.

To have an initial volume, FDK algorithm [4] can be used. Another way is to minimize the squared $\mathcal{L}_{2}$-norm between the true and the estimated projections :

$$
\boldsymbol{f}^{(0)}=\underset{f}{\arg \min }\left\{\|\boldsymbol{g}-\boldsymbol{H} \boldsymbol{f}\|^{2}\right\} .
$$

Concerning the initial labels, as in [24], $K$-means algorithm [60] can be applied to the initial volume. This is what we did for the test on simulated Shepp-Logan phantom. On the opposite, it gave very bad results for real IQI phantom. That is why, for IQI phantom, we performed a non-uniform thresholding of the initial volume. To determine the thresholds, we use the histogram of the initial volume, and we apply the peak-picking algorithm explained in [61] and originally developped for non-parametric clustering [62]. For each bin in the histogram, we seek the nearest peak in its neighbourhood : this peak is called the parent-bin of the current bin, which is so called a child-bin of this peak. Each parent-bin and its children are seen as a class, i.e. the voxels in the children-bins of parent-bin $k$ are assigned the initial class $k$. When we know the number of classes, each parent-bin is assigned as the child-bin of a higher parent-bin in its neighbourhood until there are only $K$ parents-bins.

Now, we present how to fix each parameter of the algorithm. An important one is the number of materials $K$. In industry, the number of materials in the controlled volume is known. For the simulated Shepp-Logan phantom, we can compute $K$ by using the true segmentation in figure $8:$ that gives $K=5$. For the real IQI phantom, the volume is composed of nylon, polytetrafluoroethylene (PTFE), polyamide and air : we fix $K=4$.

Next, the initialization can be used to fix several parameters thanks to their clear meaning. First, the initial volume is used to initialize the variances of the noise according to equation (37). In the initial segmentation, we compute the number of voxels $N_{k}^{(0)}$ in each class $k$. We fix the singleton energy by

$$
\alpha_{k}=\ln \left(\frac{N_{k}^{(0)}}{N}\right)
$$

Moreover, on the initial segmentation, initial means $\boldsymbol{m}^{(0)}$ and variances $\boldsymbol{v}^{(0)}$ of the classes are computed.

The parameter $m_{0}$ for the means of the prior on $\boldsymbol{m}$ is fixed so that it corresponds to an approximation of the median gray level in the volume

$$
m_{0}=\frac{1}{2}\left(\max _{j} f_{j}^{(0)}+\min _{j} f_{j}^{(0)}\right)
$$

By this way, and fixing $v_{0}$ sufficiently large, we are ensured that all possible values for $m_{k}, k \in$ $\{1, \ldots, K\}$ can be selected by the algorithm. For the same reason, parameters $\alpha_{0}$ and $\alpha_{\epsilon_{0}}$ are chosen 


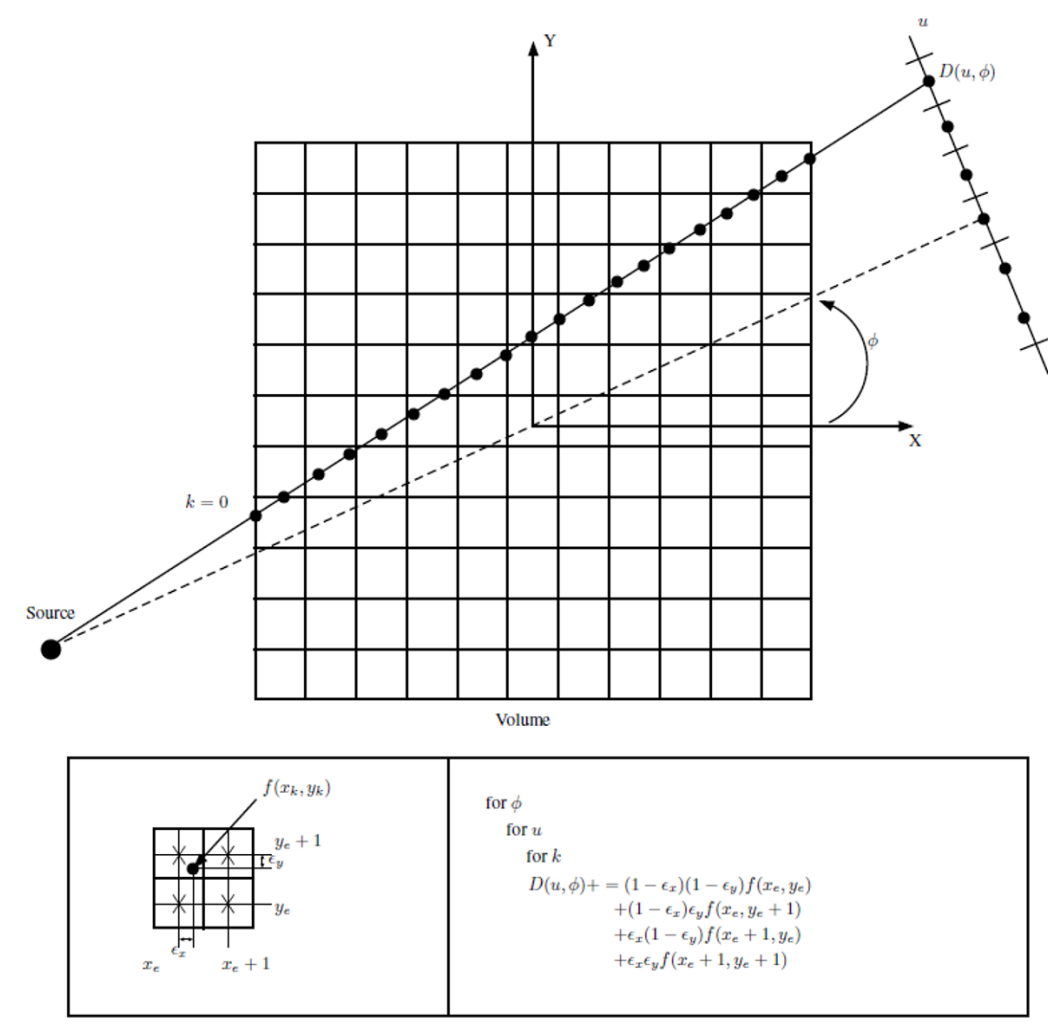

Figure 6: Ray-driven projector

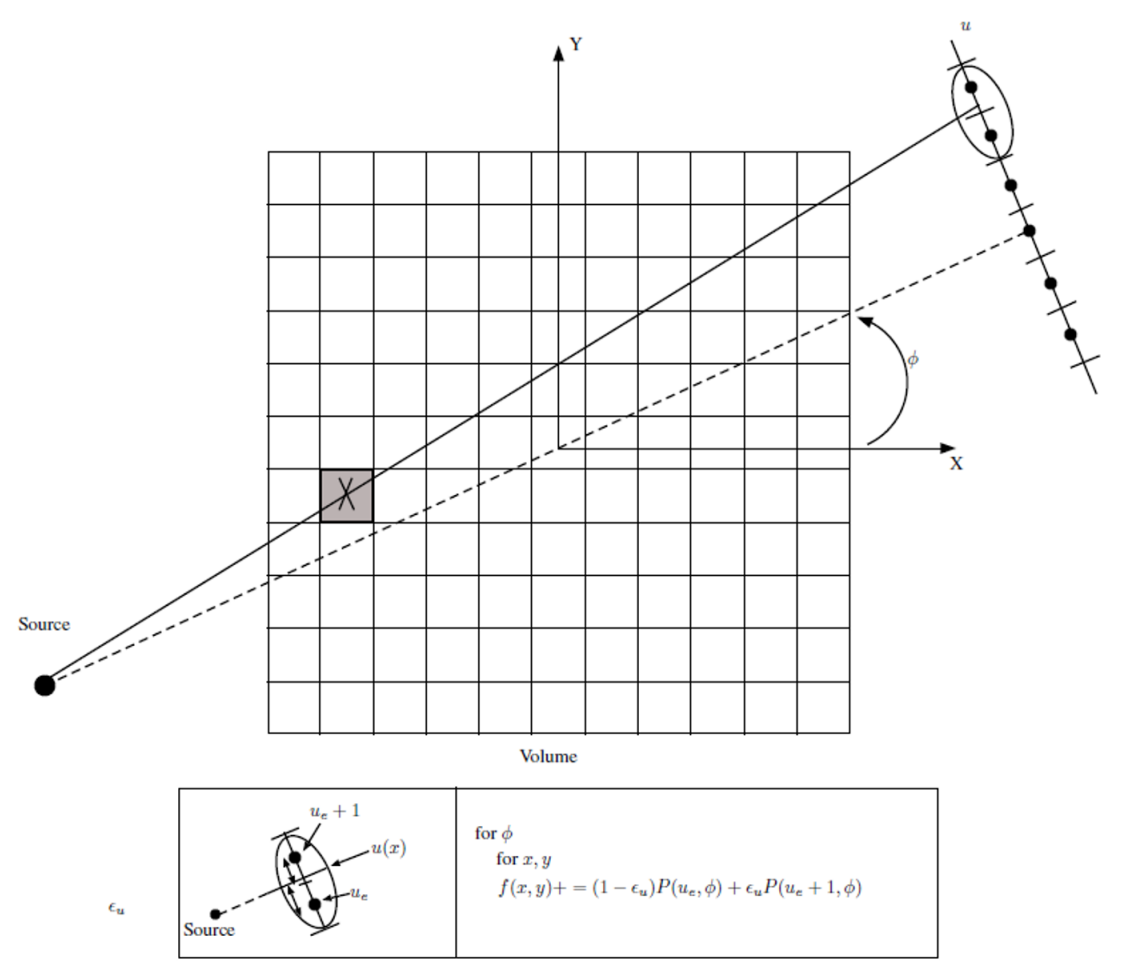

Figure 7: Voxel-driven backprojector 
sufficiently large, and $\beta_{0}$ and $\beta_{\epsilon_{0}}$ sufficiently small, so that all possible values for $v_{k}, k \in\{1, \ldots, K\}$ and $v_{\epsilon_{i}}, i \in\{1, \ldots, M\}$ are allowed. Concerning parameter $\beta_{\epsilon_{0}}$, there is a way to fix it more precisely by using a prior on signal-to-noise ratio (SNR), and using the formula

$$
\beta_{\epsilon_{0}}=\frac{\alpha_{\epsilon_{0}}-1}{M} \times\|\boldsymbol{g}\|^{2} \times \frac{10^{-\frac{S N R}{10}}}{1+10^{-\frac{S N R}{10}}} .
$$

explained in appendix B. Here, we assume $S N R=20 \mathrm{db}$.

The next parameter to fix is the granularity coefficient $\gamma_{0}$. Fixing this coefficient enforces our prior that classes in the volume are compact. So, it has to be chosen greater than the critical value $\gamma_{c}$. Because $\gamma_{c}$ is unkonwn, based on the results in figure $2, \gamma_{0}$ is fixed greater than 1 if we want to enforce compact classes. The more compact we think the classes in the volume are, the farther from $1 \gamma_{0}$ has to be fixed : here, we take $\gamma_{0}=3$. An improvement would be to fix this parameter automatically based on the initial segmentation, or at each iteration. There exist MCMC methods, like in [63] and [64], that estimate $\gamma_{0}$ based on a segmentation, without dealing with the untractable normalizing constant of $p\left(\boldsymbol{z} \mid \boldsymbol{f}, \boldsymbol{v}_{\epsilon}, \boldsymbol{m}, \boldsymbol{v} ; \boldsymbol{g}, \mathcal{M}\right)$ but, once again, a MCMC method would be too costly for our industrial application.

Tables 1 and 2 summarize the values of most of the parameters we fixed to apply our method, for reconstruction of respectively Shepp-Logan and IQI phantoms.

\begin{tabular}{|c|c|c|c|c|c|c|}
\hline Parameters & $\alpha_{\epsilon_{0}}$ & $K$ & $\gamma_{0}$ & $v_{0}$ & $\alpha_{0}$ & $\beta_{0}$ \\
\hline Fixed values & 2.1 & 5 & 3 & 1 & 5 & 0.01 \\
\hline
\end{tabular}

Table 1: Parameters of JMAP for simulated Shepp-Logan phantom reconstruction

\begin{tabular}{|c|c|c|c|c|c|c|}
\hline Parameters & $\alpha_{\epsilon_{0}}$ & $K$ & $\gamma_{0}$ & $v_{0}$ & $\alpha_{0}$ & $\beta_{0}$ \\
\hline Fixed values & 2.1 & 4 & 3 & 1 & 5 & 0.01 \\
\hline
\end{tabular}

Table 2: Parameters of JMAP for 3D IQI phantom reconstruction

\subsection{Application of the algorithm and comparison with other methods}

\subsubsection{Test on simulated data}

We first test our method on simulated Shepp-Logan phantom. Because we are in simulation, the projections are noisy such that $\mathrm{SNR}=20 \mathrm{db}$.

The initial volume is obtained by simple least-squares minimization. $K$-means algorithm [60] is applied on this volume to obtain the initial segmentation. JMAP algorithm gives the volume and the segmentation shown in figure 9. The evolution of Potts energy during the reconstruction is shown in figure 10 .

These results are compared to FDK [4] and TV minimization. To perform TV minimization, we use the algorithm described in [65], based on Bregman iterations [66]. The segmentation of FDK and TV reconstructions in figures 11 and 12 are obtained thanks to what we call a posterior Potts segmentation. This posterior Potts segmentation consider the same Gauss-Markov-Potts model we have presented here above, but with no projection operation $(\boldsymbol{H}=\boldsymbol{I}$, which is the identity matrix), on which we have performed JMAP. This model for segmentation is presented in [67]. 
Because there are too few projections compared to the size of the volume, FDK obtains very bad results. JMAP and TV reconstruct good quality volumes. The boarder between the bone and the interior of the head is sharper for TV than JMAP. On the opposite, we notice JMAP reconstruction has a high contrast while TV reconstruction is more blurred. In figure 13, we have zoomed on the above part of the middle slice of the phantom for the original and the three reconstructions. Still, we see that JMAP gives the most contrasted reconstruction, while FDK reconstruction is very noisy and TV is blurred.

In table 3, we compute the reconstruction quality indicators presented in section 4 . Because, for this phantom, the actual volume is known, we also compute

$$
\Delta_{2} f=\frac{\left\|f-f_{0}\right\|^{2}}{\left\|f_{0}\right\|^{2}}
$$

where $f_{0}$ is the true volume. Once again, the failure of FDK with few projections is highlighted. JMAP obtains the best $\Delta_{2} f$ thanks to a comprehensive prior on the volume. Concerning $C_{o m p}, d_{i s t}$ and $h_{\text {omo }}$, TV reconstruction achieves the highest values.

\begin{tabular}{|c|c|c|c|c|c|}
\hline Indicator & $\Delta_{2} \boldsymbol{g}$ & $\Delta_{2} \boldsymbol{f}$ & $C_{\text {omp }}$ & $d_{i s t}$ & $h_{\text {omo }}$ \\
\hline FDK & $167.6 \%$ & $103.1 \%$ & $84.0 \%$ & $50.4 \%$ & $49.9 \%$ \\
\hline TV & $13.5 \%$ & $26.1 \%$ & $94.2 \%$ & $81.2 \%$ & $81.0 \%$ \\
\hline JMAP & $1.35 \%$ & $18.1 \%$ & $88.7 \%$ & $68.4 \%$ & $66.8 \%$ \\
\hline
\end{tabular}

Table 3: Comparison FDK, TV and JMAP reconstruction of real IQI phantom using quality indicators defined in section 4

\subsubsection{Test on real data}

We now apply our method on an IQI phantom [53] with the parameters fixed in section 5.2. We obtain the reconstruction and the segmentation shown in figures 20 and 21. In figure 22, we show the evolution of Potts energy during the reconstruction, which converges to a maximum.

As in section 5.3.1, we compare our results on the real phantom with FDK and TV minimization. Concerning FDK, we show the reconstruction in figure 16. The corresponding segmentation is shown in figure 17. To obtain this segmentation, we performed the non-uniform thresholding based on the histogram of the volume and described in section 5.2.

These FDK reconstruction and segmentation are used as initial volume and labels for JMAP algorithm. FDK reconstruction is also used as initial volume for TV minimization algorithm. The results for this algorithm on the IQI phantom are shown in figures 18 for the reconstruction and 19 for the corresponding posterior Potts segmentation.

As we see, JMAP obtains a better quality in terms of contrast compared with both FDK and TV reconstructions. This better contrast is emphasized when in figure 23, we plot the profiles of the little holes in the bottom of the object : the profile of JMAP reconstruction is sharper than the ones of FDK and TV reconstructions. Moreover, when comparing figure 20 and 14, we can see that JMAP achieves a quality comparable to FDK reconstruction with 8 times less projections, which is a gain of time for the acquisition process.

In table 4, we compute the reconstruction quality indicators we have defined in section 4 . Because the labels for the three reconstructions are very similar, $C_{o m p}$ is practically the same for FDK, TV and JMAP. For $d_{i s t}$ and $h_{\text {omo }}$, JMAP achieves the highest values, which is in accordance with our visual evaluation. 


\begin{tabular}{|c|c|c|c|c|}
\hline Indicator & $\Delta_{2} \boldsymbol{g}$ & $C_{\text {omp }}$ & $d_{\text {ist }}$ & $h_{\text {omo }}$ \\
\hline FDK & $0.26 \%$ & $94.4 \%$ & $78.4 \%$ & $78.3 \%$ \\
\hline TV & $0.13 \%$ & $94.5 \%$ & $77.5 \%$ & $77.5 \%$ \\
\hline JMAP & $1.61 \%$ & $94.3 \%$ & $79.0 \%$ & $78.8 \%$ \\
\hline
\end{tabular}

Table 4: Comparison FDK, TV and JMAP reconstruction of real IQI phantom using quality indicators defined in section 4

\section{Conclusion and perspectives}

In this paper, we have presented a Bayesian reconstruction algorithm for 3D Computed Tomography. Developing this algorithm, the goal was to propose an enforcement of a Gauss-Markov-Potts prior model. The algorithm needed to be fast for industrial applications : that made MCMC methods unaffordable and led us to prefer JMAP algorithm.

We were also eager to propose a massively parallelizable algorithm, due to the very huge size of the volumes we are dealing with. This consideration has led us to make choices, that we have fully explained, for the estimation of each unknown in one global iteration of our method. Thanks to the prior model enforced by the algorithm, we have shown with simulated and real phantoms that our method achieves high quality reconstructions which outperform FDK and Total Variation minimization. Another great advantage is our method returns labels of voxels jointly with the reconstructed volume. Moreover, thanks to the fact that the prior model is clearly expressed in terms of probability distributions, tuning the parameters of our algorithm is very straightforward, based on the strategy we have detailed in section 5.2. For Total Variation minimization algorithm, this step has been more difficult, because the meaning of its parameters is not so clear.

In addition, we have investigated some reconstruction quality indicators in order to quatitatively compare FDK, TV and JMAP algorithms. These indicators have been useful to fully analyze our results in terms of compacity, homogeneity and distinguishability of the regions in the reconstructed volumes.

A future work would be to implement a full-GPU version of the method. A proof of convergence of the algorithm, with conditions on the initialization and the parameters, would be also valuable. A work on Variational Bayesian Approach on this model in the same context is also currently underway.

\section{A. Simple Gradient descent to estimate the object $f$ in JMAP algorithm}

In section 3.2, the criterion to minimize in order to estimate the object in one iteration of JMAP is

$$
J(\boldsymbol{f})=\|\boldsymbol{g}-\boldsymbol{H} \boldsymbol{f}\|_{\boldsymbol{V}_{\epsilon}}^{2}+\left\|\boldsymbol{f}-\boldsymbol{m}_{\boldsymbol{z}}\right\|_{\boldsymbol{V}_{\boldsymbol{z}}}^{2} .
$$

We do that by a simple gradient descent, that we detail in this section.

At iteration $(k+1)$ of simple gradient descent, we update the object as follows :

$$
\boldsymbol{f}^{(k+1)}=\boldsymbol{f}^{(k)}-\lambda_{k} \nabla J\left(\boldsymbol{f}^{(k)}\right)
$$

where

$$
\lambda_{k}=\underset{\lambda>0}{\arg \min }\left\{J\left(\boldsymbol{f}^{(k+1)}\right)\right\}
$$

is the optimal step of the descent. The gradient reads

$$
\nabla J(\boldsymbol{f})=2\left(\boldsymbol{H}^{T} \boldsymbol{V}_{\epsilon}^{-1} \boldsymbol{H}+\boldsymbol{V}_{\boldsymbol{z}}^{-1}\right) \boldsymbol{f}-2\left(\boldsymbol{V}_{\boldsymbol{z}}^{-1} \boldsymbol{m}_{\boldsymbol{z}}+\boldsymbol{H}^{T} \boldsymbol{V}_{\epsilon}^{-1} \boldsymbol{g}\right)
$$




$$
\Rightarrow \nabla J(\boldsymbol{f})=2 \boldsymbol{H}^{T} \boldsymbol{V}_{\epsilon}^{-1}(\boldsymbol{H} \boldsymbol{f}-\boldsymbol{g})+2 \boldsymbol{V}_{\boldsymbol{z}}^{-1}\left(\boldsymbol{f}-\boldsymbol{m}_{\boldsymbol{z}}\right)
$$

As we see, the computation of the gradient requires one projection ( $\boldsymbol{H}$ operator), which is already done when we compute the criterion (see (27)), and one backprojection $\left(\boldsymbol{H}^{T}\right.$ operator).

To find the optimal step $\lambda_{k}$, we compute

$$
\begin{aligned}
J\left(\boldsymbol{f}^{(k+1)}\right)= & J\left(\boldsymbol{f}^{(k)}\right)+\lambda_{k}^{2} \nabla J\left(\boldsymbol{f}^{(k)}\right)^{T}\left[\boldsymbol{V}_{\boldsymbol{z}}^{-1}+\boldsymbol{H}^{T} \boldsymbol{V}_{\epsilon}^{-1} \boldsymbol{H}\right] \nabla J\left(\boldsymbol{f}^{(k)}\right) \\
& +2 \lambda_{k}\left[\left(\boldsymbol{g}-\boldsymbol{H} \boldsymbol{f}^{(k)}\right)^{T} \boldsymbol{V}_{\boldsymbol{\epsilon}}^{-1} \boldsymbol{H}-\left(\boldsymbol{f}^{(k)}-\boldsymbol{m}_{\boldsymbol{z}}\right)^{T} \boldsymbol{V}_{\boldsymbol{z}}^{-1}\right] \nabla J\left(\boldsymbol{f}^{(k)}\right)
\end{aligned}
$$

and we solve

$$
\frac{\mathrm{d} J\left(\boldsymbol{f}^{(k+1)}\right)}{\mathrm{d} \lambda_{k}}=0
$$

which leads to :

$$
\lambda_{k}=\frac{\left\|\nabla J\left(\boldsymbol{f}^{(k)}\right)\right\|^{2}}{2 \nabla J\left(\boldsymbol{f}^{(k)}\right)^{T}\left[\boldsymbol{V}_{\boldsymbol{z}}^{-1}+\boldsymbol{H}^{T} \boldsymbol{V}_{\epsilon}^{-1} \boldsymbol{H}\right] \nabla J\left(\boldsymbol{f}^{(k)}\right)}
$$

which can be rewritten :

$$
\lambda_{k}=\frac{\left\|\nabla J\left(\boldsymbol{f}^{(k)}\right)\right\|^{2}}{2\left[\left\|\boldsymbol{V}_{\boldsymbol{z}}^{-\frac{1}{2}} \nabla J\left(\boldsymbol{f}^{(k)}\right)\right\|^{2}+\left\|\boldsymbol{V}_{\epsilon}^{-\frac{1}{2}} \boldsymbol{H} \nabla J\left(\boldsymbol{f}^{(k)}\right)\right\|^{2}\right]}
$$

The computation of $\lambda_{k}$ requires one projection of the gradient $\nabla J\left(\boldsymbol{f}^{(k)}\right)$. As stop criterion, we use the relative evolution of the criterion $J(\boldsymbol{f})$ between two iterations :

$$
\left|\frac{J\left(\boldsymbol{f}^{(k+1)}\right)-J\left(\boldsymbol{f}^{(k)}\right)}{J\left(\boldsymbol{f}^{(k)}\right)}\right|=\frac{1}{2} \frac{\left\|\nabla J\left(\boldsymbol{f}^{(k)}\right)\right\|^{2}\left|\lambda_{k}\right|}{\left|J\left(\boldsymbol{f}^{(k)}\right)\right|}
$$

\section{B. Parameters for the prior of the variances of the noise}

The prior of the variances of the noise $\epsilon$ is

$$
p\left(v_{\epsilon_{i}} \mid \alpha_{\epsilon_{0}}, \beta_{\epsilon_{0}}\right)=\mathcal{I} \mathcal{G}\left(v_{\epsilon_{i}} \mid \alpha_{\epsilon_{0}}, \beta_{\epsilon_{0}}\right), \forall i
$$

We fix $\alpha_{\epsilon_{0}}$. Next, we want to fix $\beta_{\epsilon_{0}}$ with respect to $\alpha_{\epsilon_{0}}$ and SNR, in order to perform reconstruction using JMAP algorithm explained in section 3. SNR (Signal-to-Noise Ratio) is defined by

$$
S N R=10 \log \left(\frac{\left\|\boldsymbol{g}_{0}\right\|^{2}}{\|\boldsymbol{\epsilon}\|^{2}}\right)
$$

where $\log$ is the logarithm to the base 10 , and $\boldsymbol{g}_{0}$ the unnoisy projections of the object. Because we do not know these unnoisy projections, we make the common assumption that the noise $\epsilon$ and the unnoisy projections $\boldsymbol{g}_{0}$ are orthogonal :

$$
\|\boldsymbol{g}\|^{2}=\left\|\boldsymbol{g}_{0}\right\|^{2}+\|\boldsymbol{\epsilon}\|^{2}
$$

That leads to

$$
S N R=10 \log \left(\frac{\|\boldsymbol{g}\|^{2}}{\|\boldsymbol{\epsilon}\|^{2}}-1\right)
$$




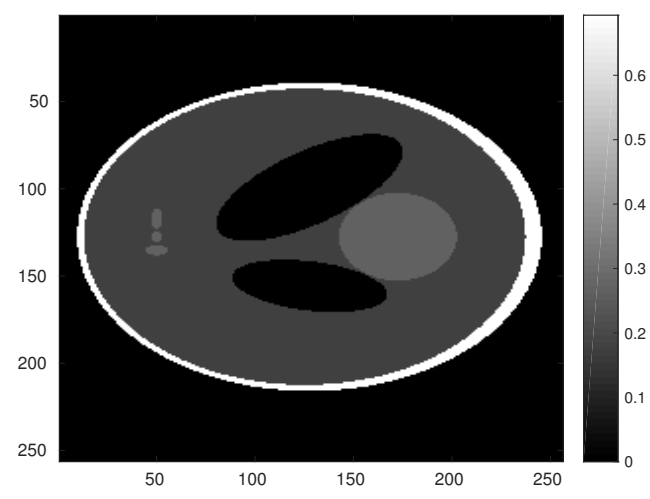

(a)

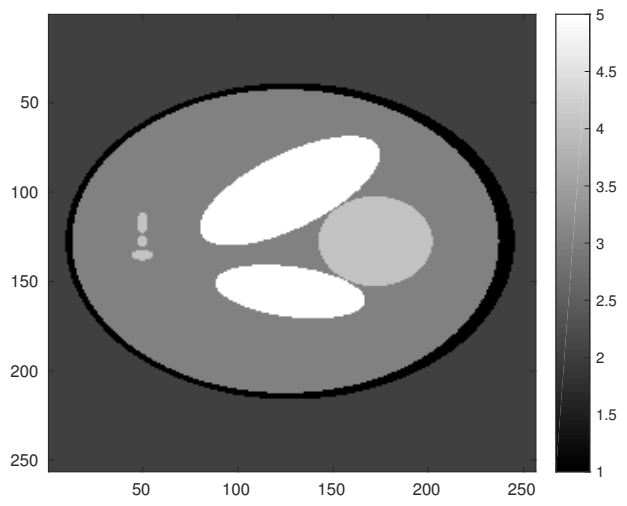

(b)

Figure 8: Shepp-Logan phantom (a) and its segmentation in $K=5$ classes (b) (middle slice)

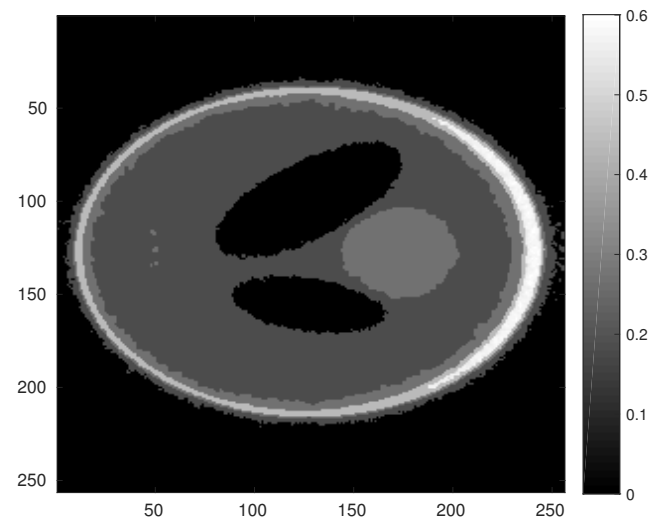

(a)

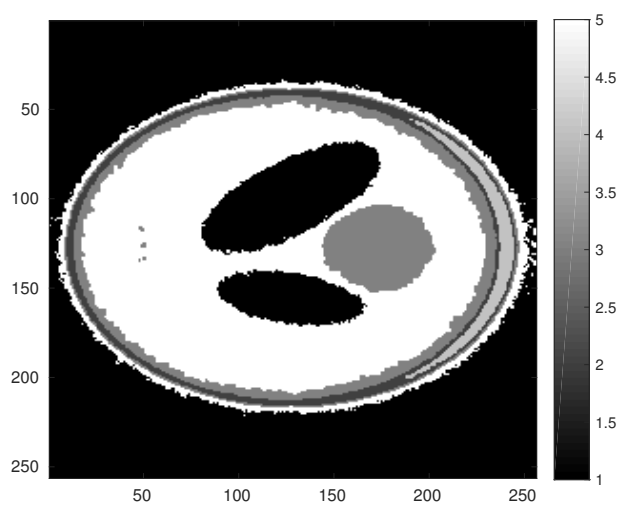

(b)

Figure 9: Jointly retrieved reconstruction (a) and segmentation in $K=5$ classes (b) of Shepp-Logan phantom (middle slice)

We approximate $\|\boldsymbol{\epsilon}\|^{2}$ by its expectation, which gives :

$$
\begin{aligned}
\|\boldsymbol{\epsilon}\|^{2} \approx \mathbb{E}\left(\boldsymbol{\epsilon}^{T} \boldsymbol{\epsilon}\right) & =\operatorname{Tr}\left[\mathbb{E}\left(\boldsymbol{\epsilon} \boldsymbol{\epsilon}^{T}\right)\right] \\
& =\operatorname{Tr}\left[\mathbb{E}\left(\mathbb{E}\left(\boldsymbol{\epsilon} \boldsymbol{\epsilon}^{T} \mid \boldsymbol{V}_{\epsilon}\right)\right)\right] \\
& =\operatorname{Tr}\left[\mathbb{E}\left(\boldsymbol{V}_{\epsilon}\right)\right] \\
& =\mathbb{E}\left(\operatorname{Tr}\left[\boldsymbol{V}_{\epsilon}\right]\right) \\
& =\mathbb{E}\left(\sum_{i=1}^{M} v_{\epsilon_{i}}\right) \\
& =\sum_{i=1}^{M} \mathbb{E}\left(v_{\epsilon_{i}}\right) \\
& =M \times \frac{\beta_{\epsilon_{0}}}{\left(\alpha_{\epsilon_{0}}-1\right)}
\end{aligned}
$$

Using the definition of SNR in equation (62), we find the expression given in equation (53) to fix $\beta_{\epsilon_{0}}$

$$
\beta_{\epsilon_{0}}=\frac{\alpha_{\epsilon_{0}}-1}{M} \times\|\boldsymbol{g}\|^{2} \times \frac{10^{-\frac{S N R}{10}}}{1+10^{-\frac{S N R}{10}}} .
$$




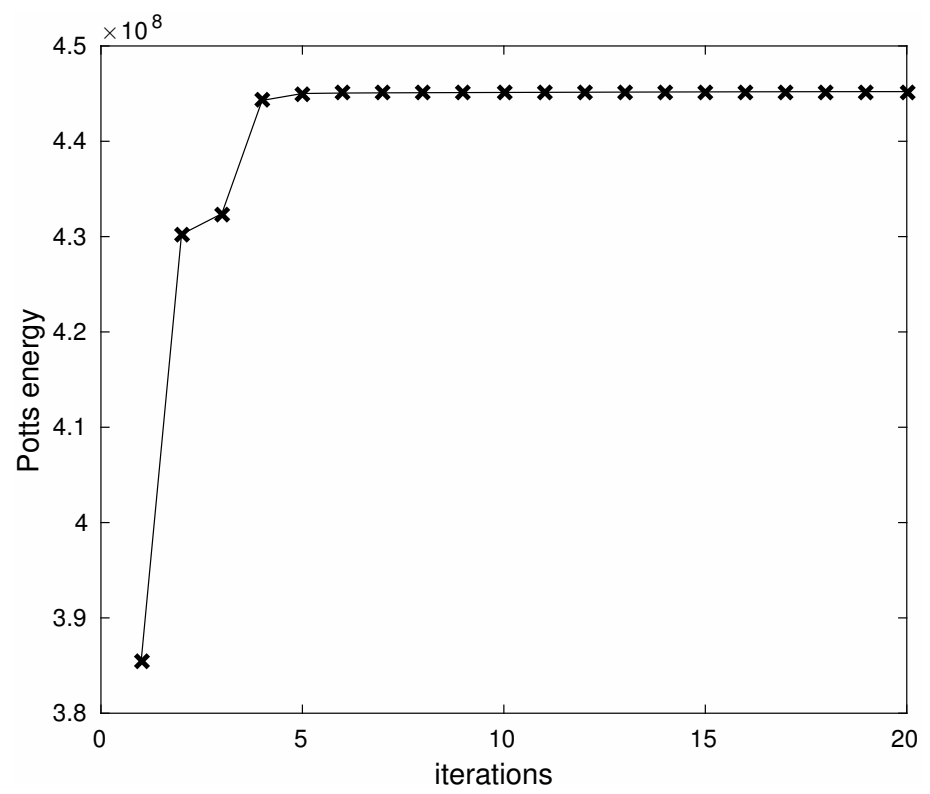

Figure 10: Evolution of Potts energy during JMAP reconstruction of Shepp-Logen phantom

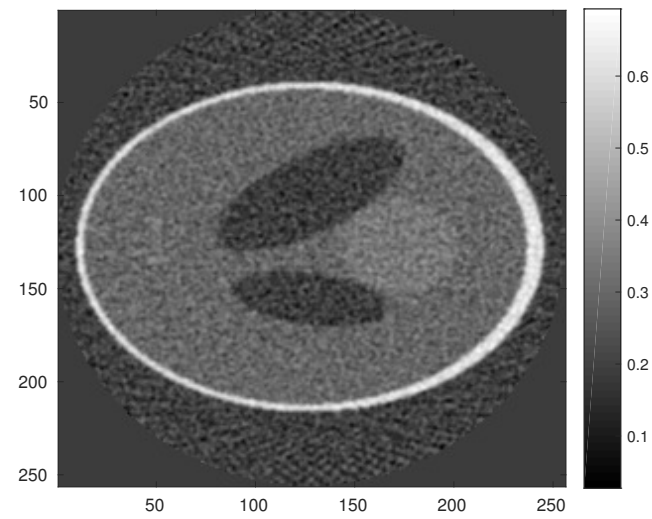

(a)

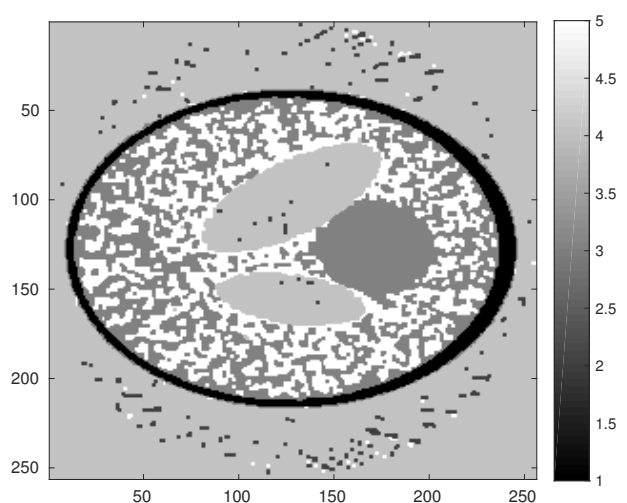

(b)

Figure 11: FDK reconstruction (a) and posterior segmentation in $K=5$ classes (b) of Shepp-Logan phantom (middle slice)

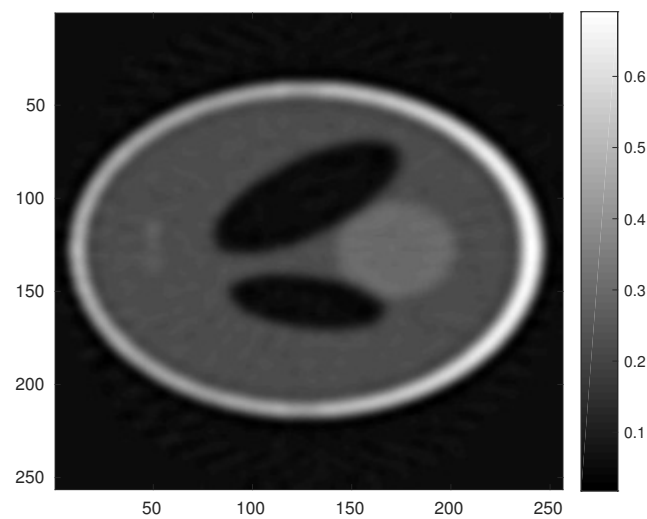

(a)

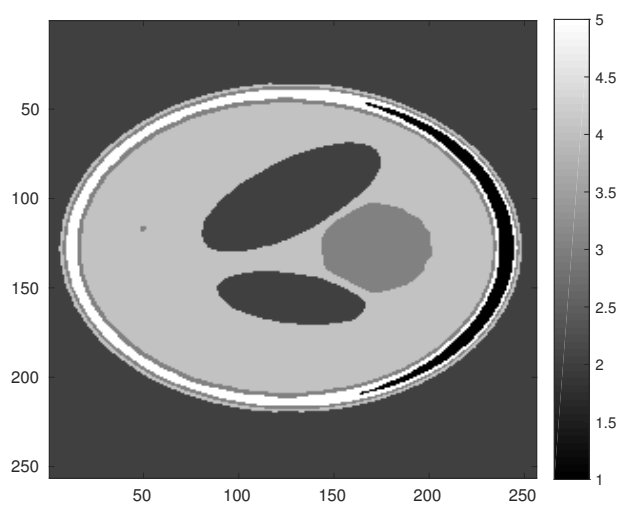

(b)

Figure 12: TV reconstruction (a) and posterior segmentation in $K=5$ classes (b) of Shepp-Logan phantom (middle slice) 


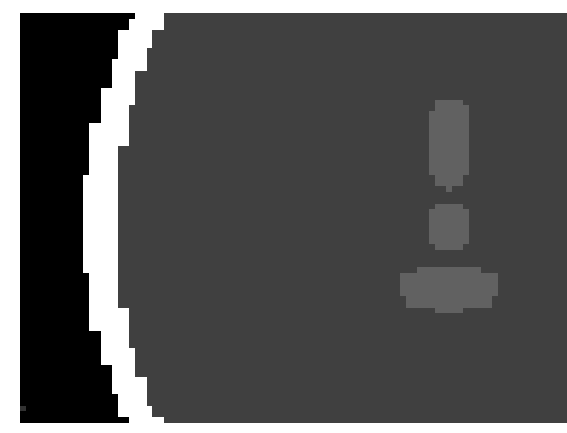

(a)

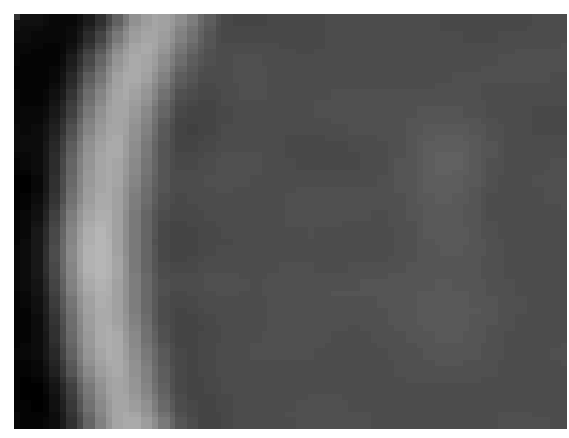

(c)

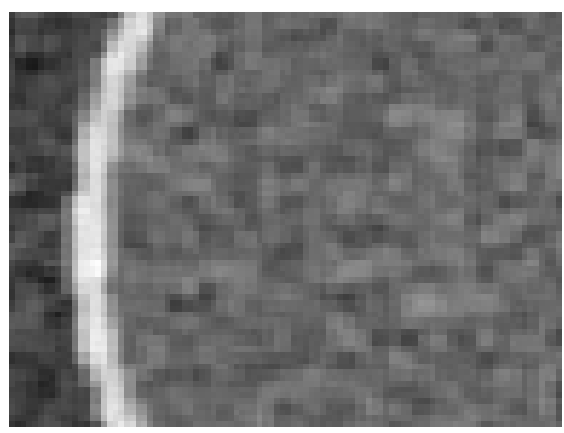

(b)

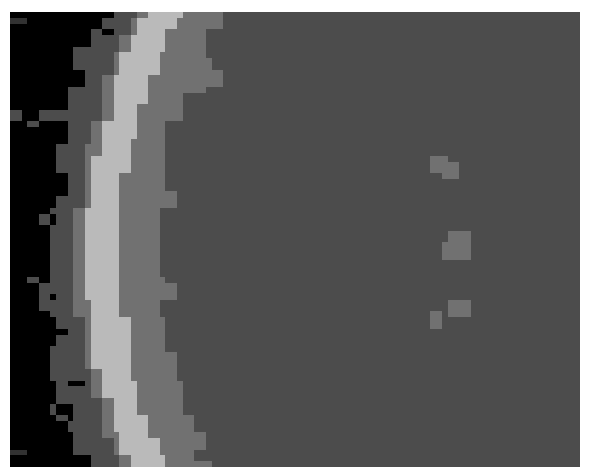

(d)

Figure 13: Zooms on original Shepp-Logan phantom (a), and its reconstructions by FDK (b), TV (c) and JMAP (d)
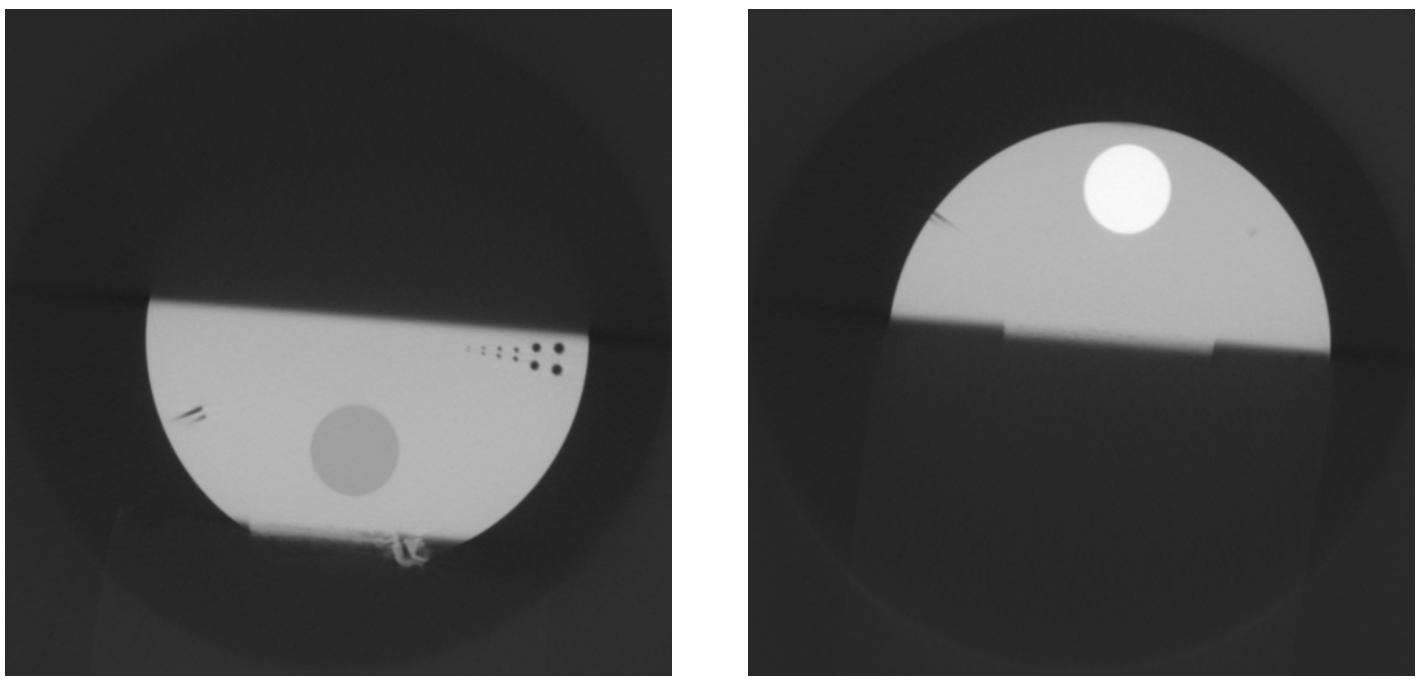

Figure 14: Real IQI phantom, obtained by FDK from 2400 projections (bottom and top) 


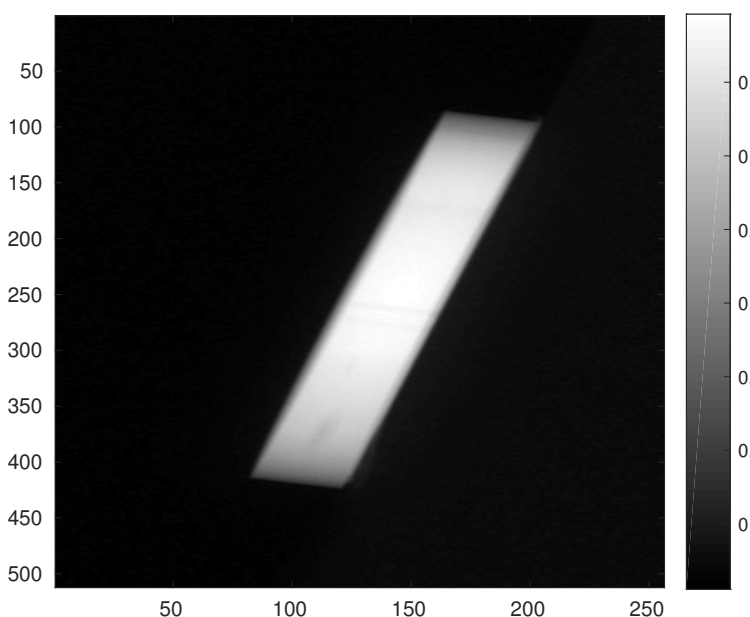

(a)

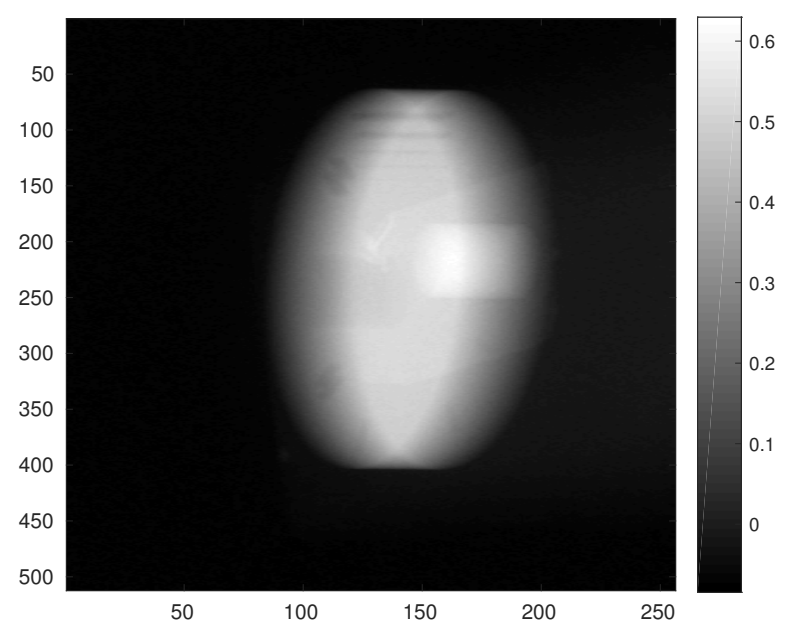

(b)

Figure 15: Two projections at angle $0^{\circ}$ (a) and $90^{\circ}$ (b) of the volume to reconstruct

\section{References}

[1] Radon J. 1.1 Über die bestimmung von funktionen durch ihre integralwerte längs gewisser mannigfaltigkeiten. Classic papers in modern diagnostic radiology. 2005;5.

[2] Smith KT, Keinert F. Mathematical foundations of computed tomography. Applied Optics. 1985;24(23):3950-3957.

[3] Rodet T. Algorithmes rapides de reconstruction en tomographie par compression des calculs: application à la tomofluoroscopie 3D. Grenoble, INPG; 2002.

[4] Feldkamp L, Davis L, Kress J. Practical cone-beam algorithm. JOSA A. 1984;1(6):612-619.

[5] Katsevich A. Theoretically exact filtered backprojection-type inversion algorithm for spiral CT. SIAM Journal on Applied Mathematics. 2002;62(6):2012-2026.

[6] Katsevich A. Analysis of an exact inversion algorithm for spiral cone-beam CT. Physics in medicine and biology. 2002;47(15):2583.

[7] Kudo H, Rodet T, Noo F, Defrise M. Exact and approximate algorithms for helical cone-beam CT. Physics in medicine and biology. 2004;49(13):2913.

[8] De Rosier D, Klug A. Reconstruction of three dimensional structures from electron micrographs. Nature. 1968;217(5124):130-134.

[9] Jackson JI, Meyer CH, Nishimura DG, Macovski A. Selection of a convolution function for Fourier inversion using gridding [computerized tomography application]. Medical Imaging, IEEE Transactions on. 1991;10(3):473-478.

[10] Gordon R, Bender R, Herman GT. Algebraic reconstruction techniques (ART) for threedimensional electron microscopy and X-ray photography. Journal of theoretical Biology. 1970;29(3):471-481.

[11] Idier J. Approche bayésienne pour les problèmes inverses. Hermès Science Publications; 2001.

[12] Gilbert P. Iterative methods for the three-dimensional reconstruction of an object from projections. Journal of theoretical biology. 1972;36(1):105-117.

[13] Andersen AH, Kak AC. Simultaneous algebraic reconstruction technique (SART): a superior implementation of the ART algorithm. Ultrasonic imaging. 1984;6(1):81-94. 

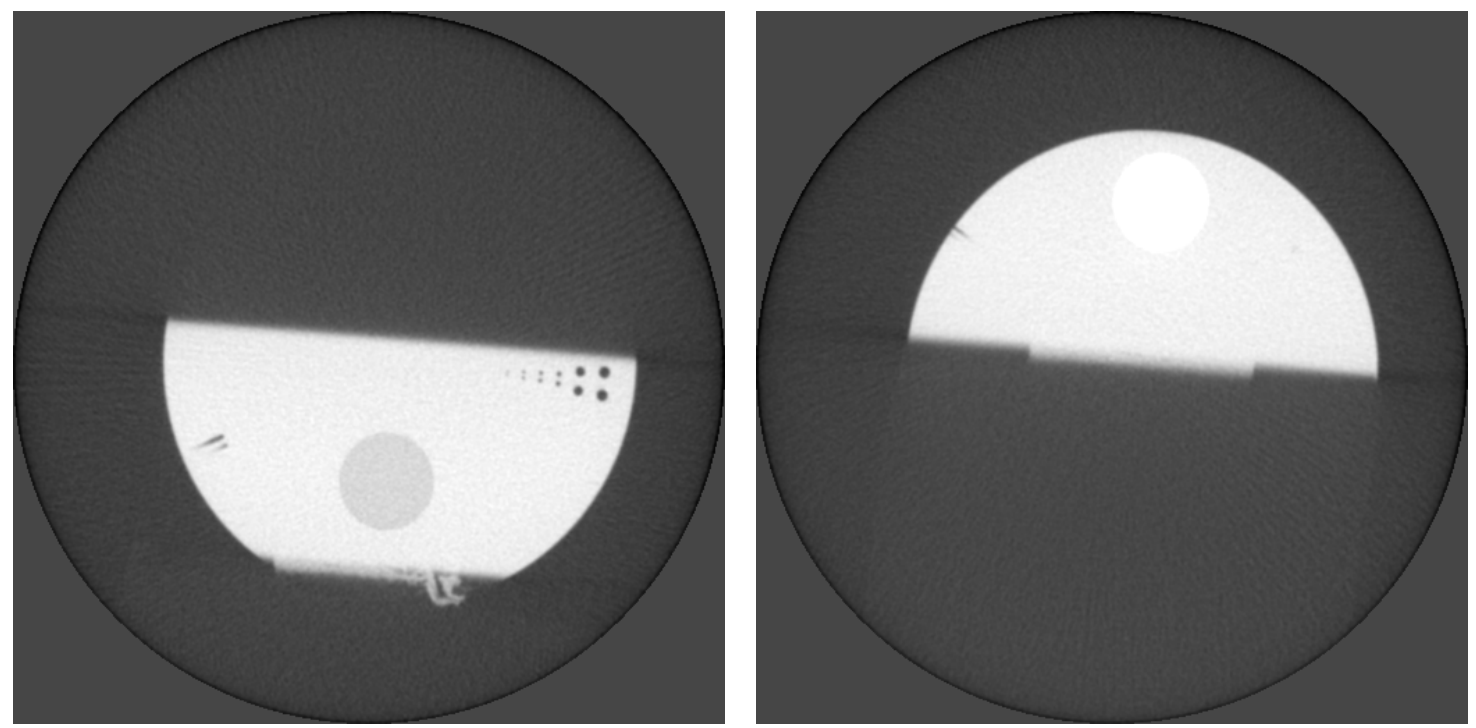

Figure 16: FDK reconstruction (bottom and top) from 300 projections
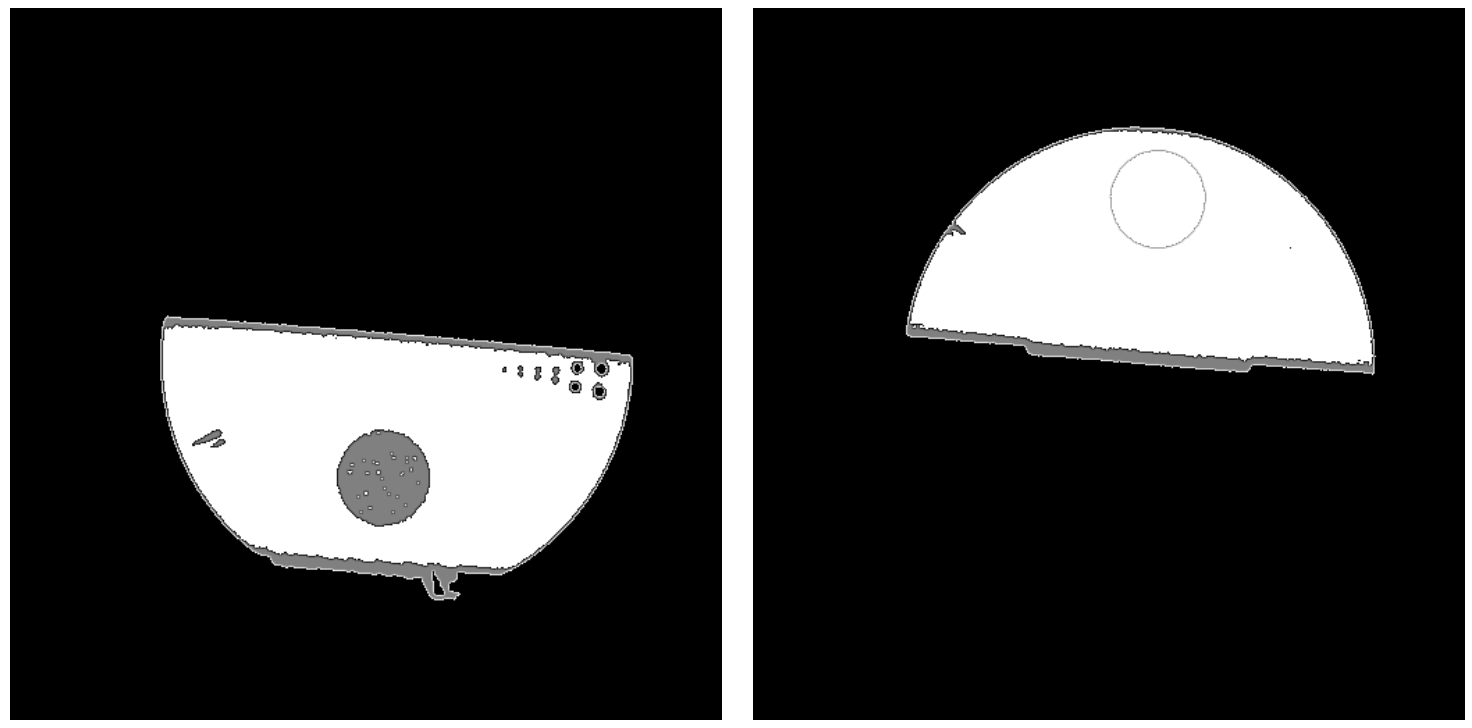

Figure 17: Segmentation of FDK reconstruction (bottom and top) with 300 projections 

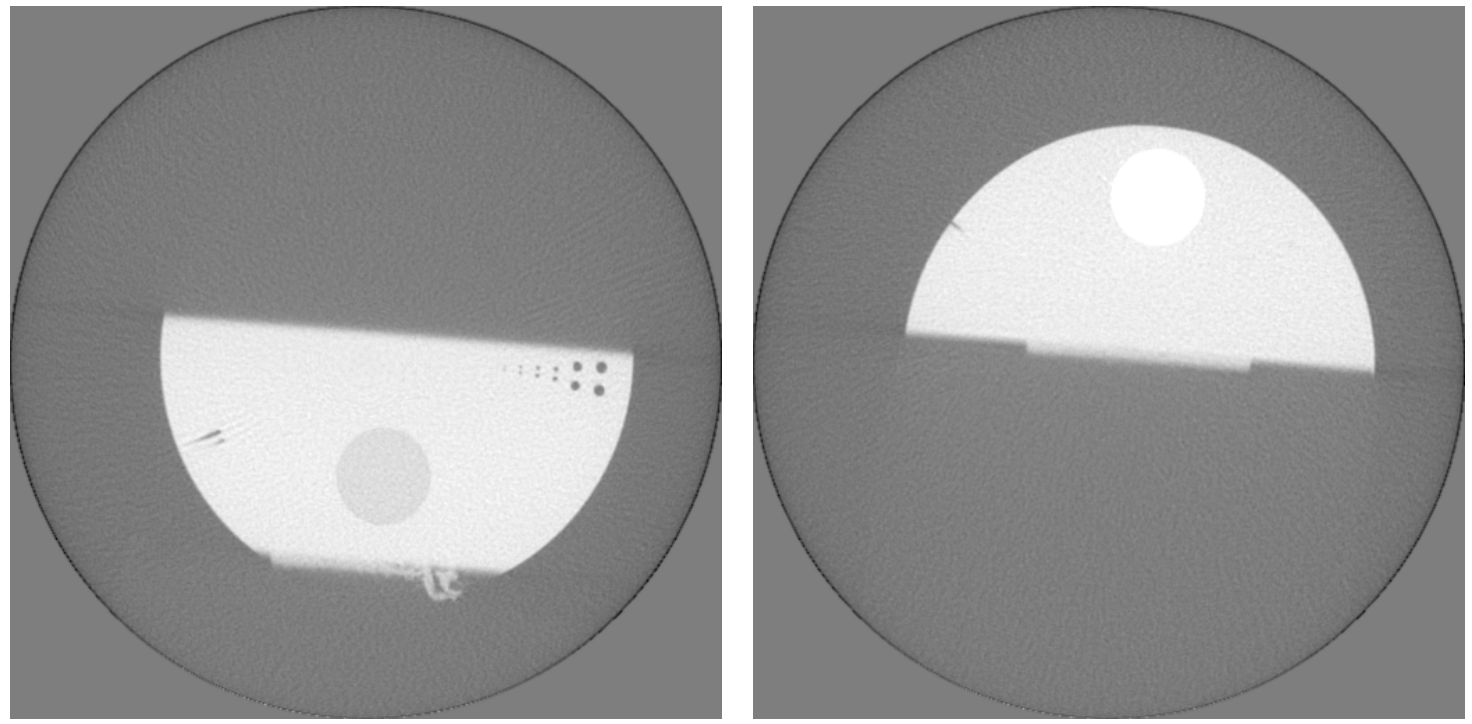

Figure 18: TV reconstruction (bottom and top) from 300 projections
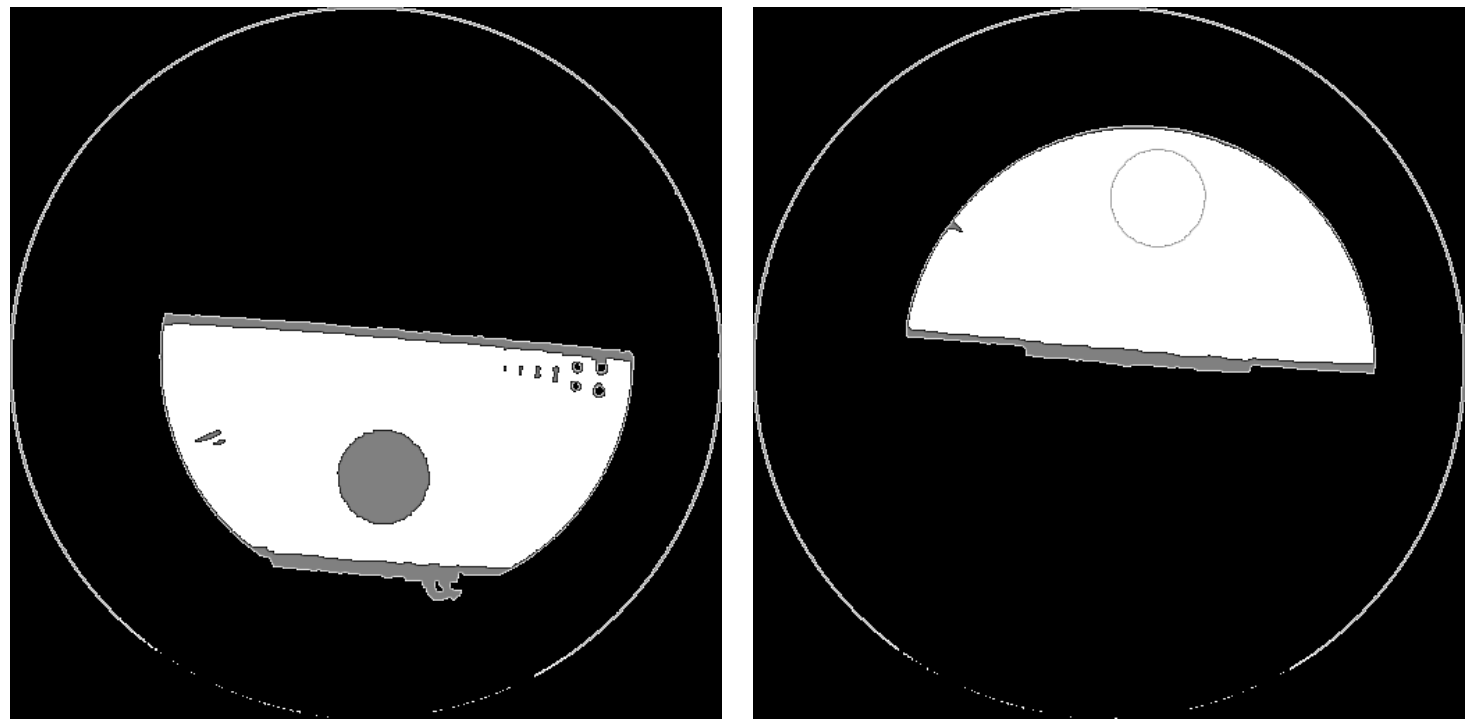

Figure 19: Segmentation of TV reconstruction (bottom and top) with 300 projections 

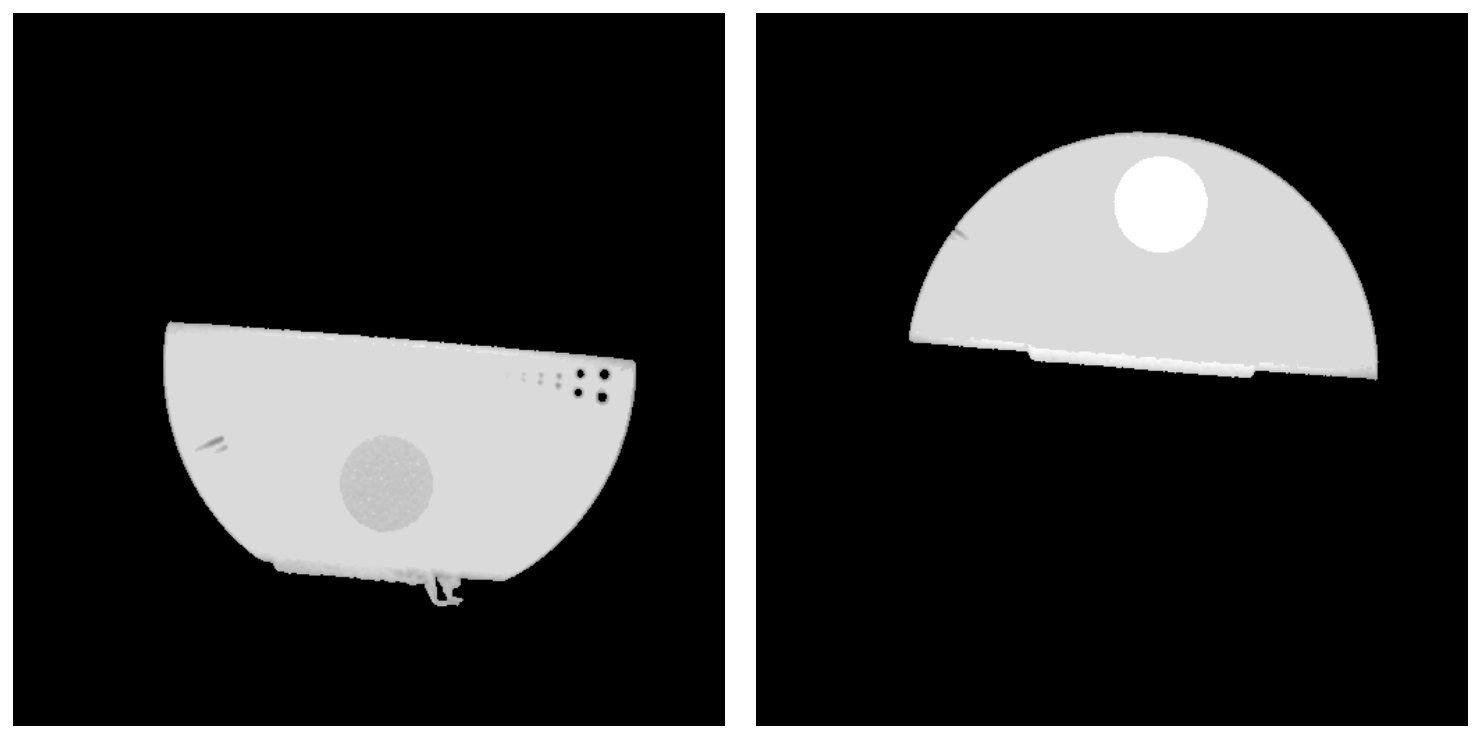

Figure 20: Reconstruction obtained by JMAP (bottom and top) from 300 projections
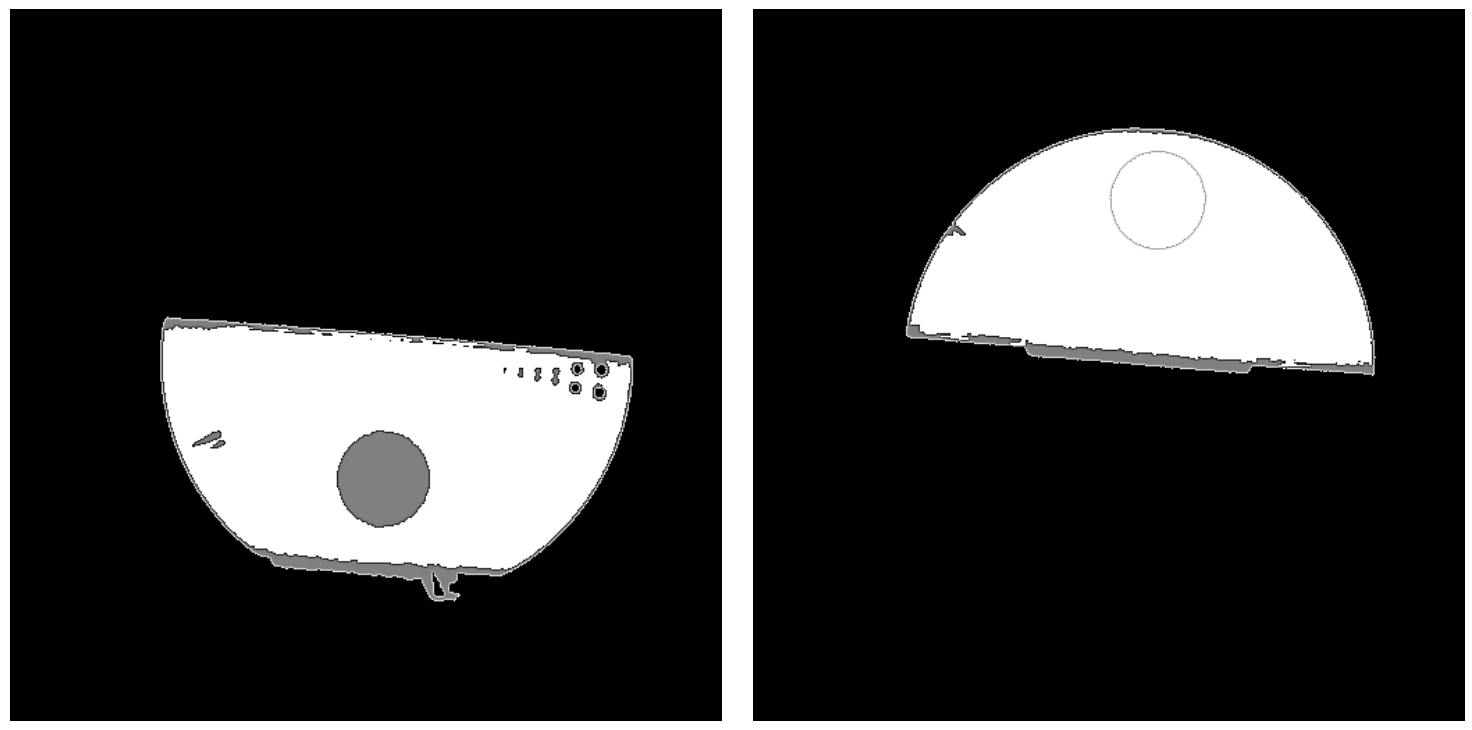

Figure 21: Segmentation obtained by JMAP (bottom and top) from 300 projections 


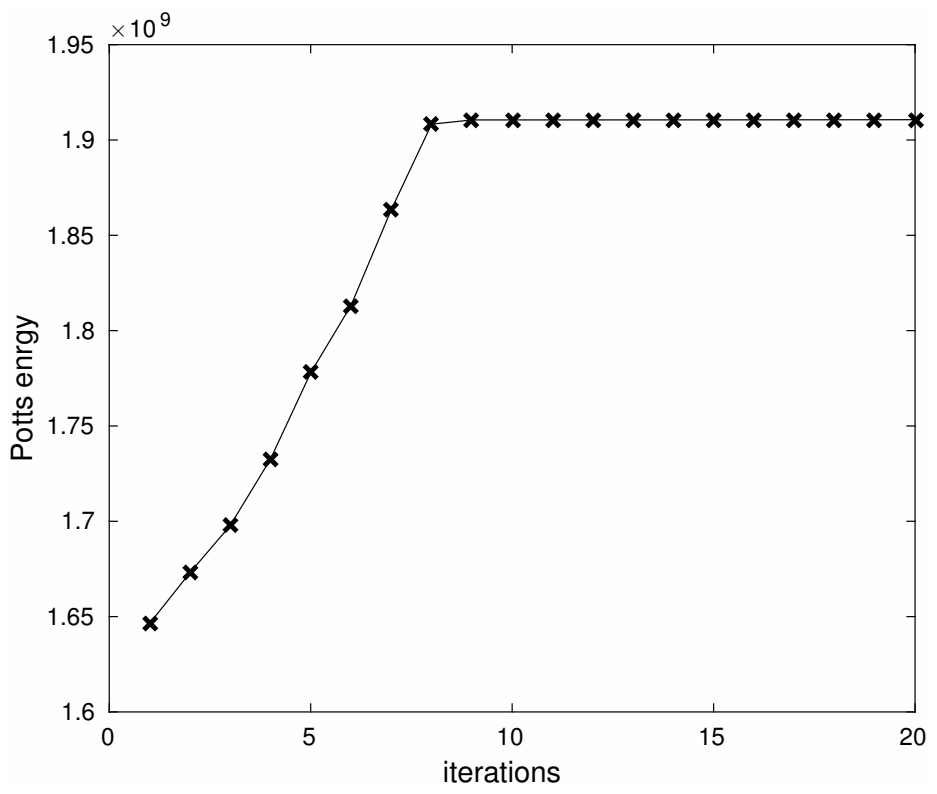

Figure 22: Evolution of Potts energy during JMAP reconstruction of real IQI phantom

[14] Mohammad-Djafari A, Demoment G. Maximum entropy image reconstruction in X-ray and diffraction tomography. Medical Imaging, IEEE Transactions on. 1988;7(4):345-354.

[15] Gac N, Vabre A, Mohammad-Djafari A, Rabanal A, Buyens F. GPU implementation of a 3D bayesian CT algorithm and its application on real foam reconstruction. In: The First International Conference on Image Formation in X-Ray Computed Tomography; 2010. p. 151-155.

[16] Batenburg KJ, Sijbers J. DART: a practical reconstruction algorithm for discrete tomography. Image Processing, IEEE Transactions on. 2011;20(9):2542-2553.

[17] Bleichrodt F. Improving robustness of tomographic reconstruction methods. Mathematical Institute, Faculty of Science, Leiden University; 2015.

[18] Sidky EY, Jakob H, Pan X, et al. Convex optimization problem prototyping for image reconstruction in computed tomography with the Chambolle \& Pock algorithm. Physics in medicine and biology. 2012;57(10):3065.

[19] Chambolle A, Pock T. A first-order primal-dual algorithm for convex problems with applications to imaging. Journal of Mathematical Imaging and Vision. 2011;40(1):120-145.

[20] Mumford D, Shah J. Optimal approximations by piecewise smooth functions and associated variational problems. Communications on pure and applied mathematics. 1989;42(5):577-685.

[21] Storath M, Weinmann A, Frikel J, Unser M. Joint image reconstruction and segmentation using the Potts model. Inverse Problems. 2015;31(2):025003.

[22] Féron O, Duchêne B, Mohammad-Djafari A. Microwave imaging of inhomogeneous objects made of a finite number of dielectric and conductive materials from experimental data. Inverse Problems. 2005;21(6):S95.

[23] Féron O. Champs de Markov cachés pour les problèmes inverses. Application à la fusion de données et à la reconstruction dimages en tomographie micro-onde. Thèse de Doctorat, Université de Paris-Sud, Orsay; 2006.

[24] Ayasso H. Une approche bayésienne de l'inversion. Application à l'imagerie de diffraction dans les domaines micro-onde et optique. Université Paris Sud-Paris XI; 2010.

[25] Ayasso H, Mohammad-Djafari A. Joint NDT image restoration and segmentation using GaussMarkov-Potts prior models and variational bayesian computation. Image Processing, IEEE Transactions on. 2010;19(9):2265-2277. 


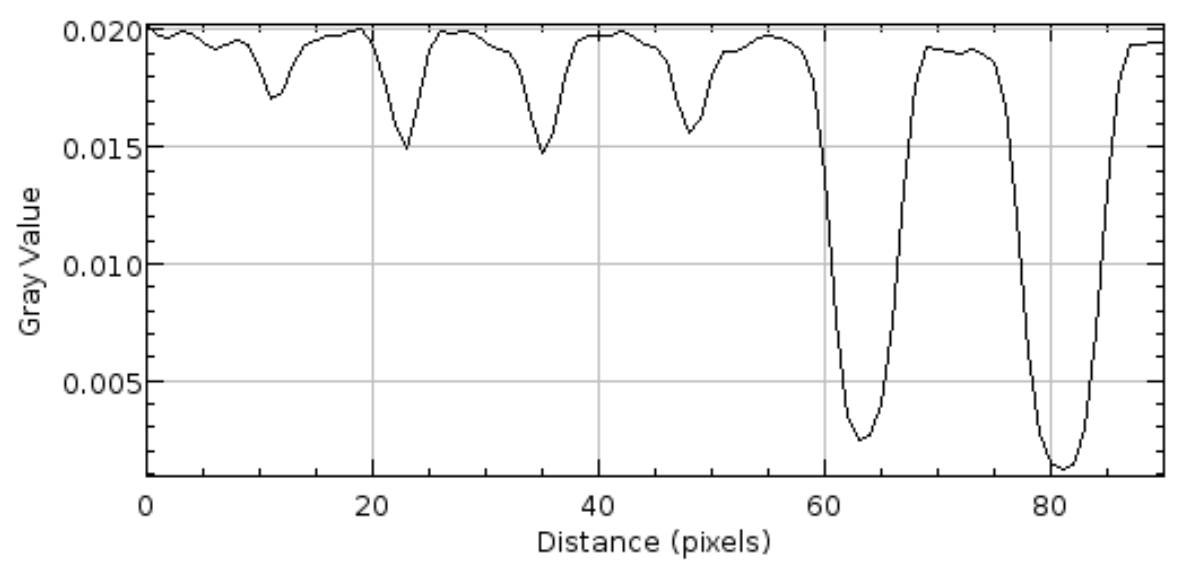

(a)

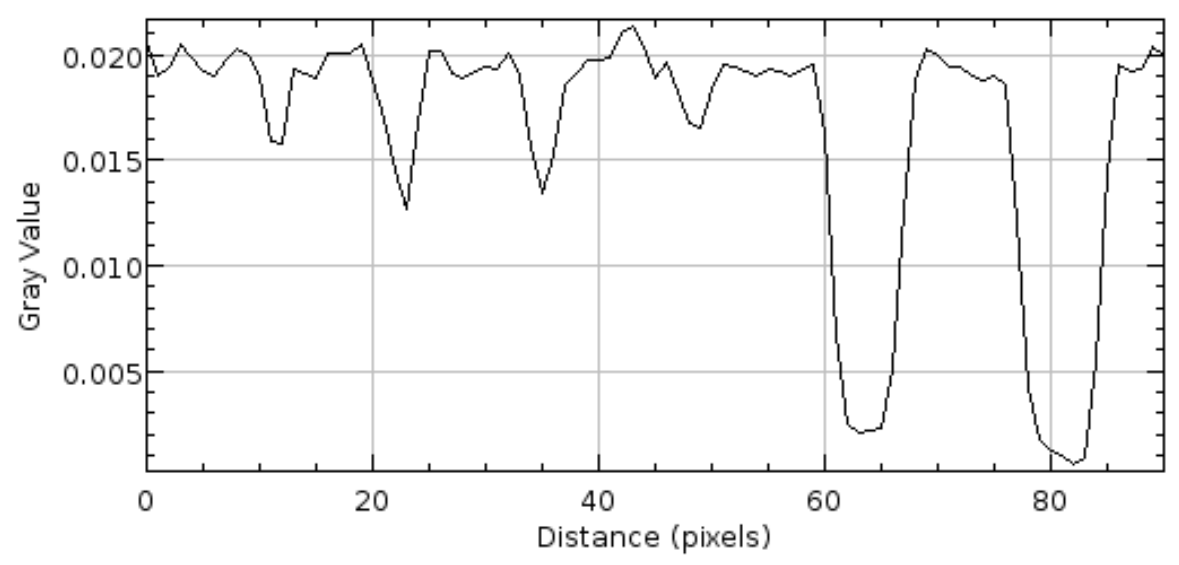

(b)

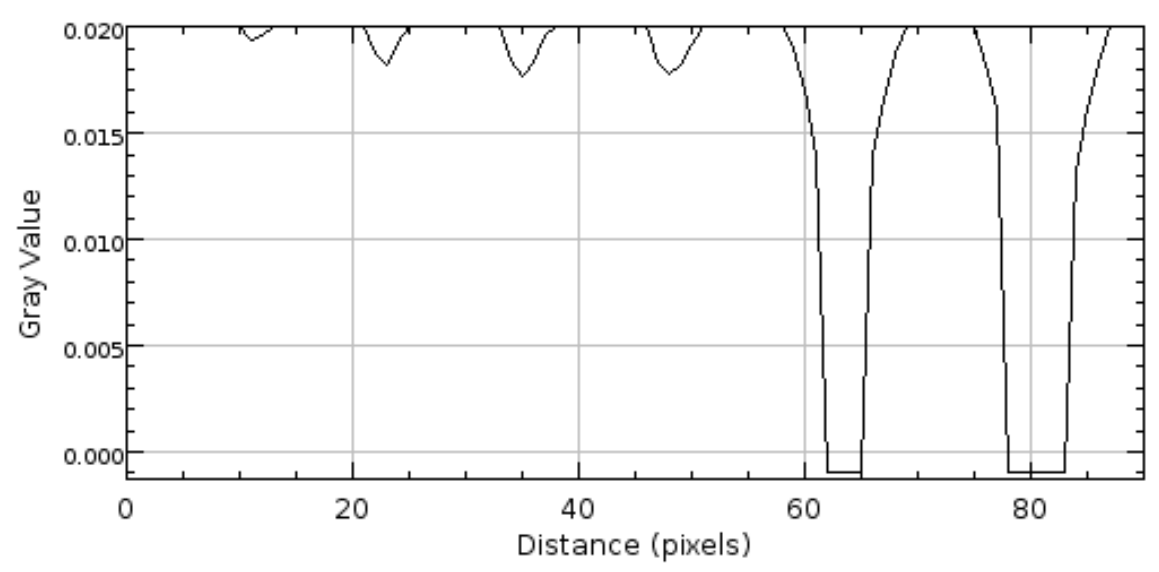

(c)

Figure 23: Profiles of holes for FDK (a), TV (b) and JMAP (c) reconstructions of real IQI phantom from 300 projections 
[26] Ayasso H, Duchêne B, Mohammad-Djafari A. Bayesian inversion for optical diffraction tomography. Journal of Modern Optics. 2010;57(9):765-776.

[27] Dumitru M. Approche bayésienne de l'estimation des composantes périodiques des signaux en chronobiologie. Paris Saclay; 2016.

[28] Onsager L. Crystal statistics. I. A two-dimensional model with an order-disorder transition. Physical Review. 1944;65(3-4):117.

[29] Huang K. Statistical mechanics. Wiley; 1987.

[30] Giovannelli JF. Estimation of the Ising field parameter thanks to the exact partition function. In: ICIP; 2010. p. 1441-1444.

[31] Mohammad-Djafari A, Ayasso H. Variational Bayes and mean field approximations for Markov field unsupervised estimation. In: 2009 IEEE International Workshop on Machine Learning for Signal Processing. IEEE; 2009. p. 1-6.

[32] Pereyra M, Schniter P, Chouzenoux E, Pesquet JC, Tourneret JY, Hero AO, et al. A survey of stochastic simulation and optimization methods in signal processing. IEEE Journal of Selected Topics in Signal Processing. 2016;10(2):224-241.

[33] Zhang J. The mean field theory in EM procedures for Markov random fields. IEEE Transactions on signal processing. 1992;40(10):2570-2583.

[34] Zhang J. The mean field theory in EM procedures for blind Markov random field image restoration. IEEE Transactions on Image Processing. 1993;2(1):27-40.

[35] Zhao N, Basarab A, Kouame D, Tourneret JY. Joint segmentation and deconvolution of ultrasound images using a hierarchical Bayesian model based on generalized Gaussian priors. IEEE transactions on Image Processing. 2016;25(8):3736-3750.

[36] Féron O, Orieux F, Giovannelli JF. Echantillonnage de champs gaussiens de grande dimension. In: 42èmes Journées de Statistique; 2010. .

[37] Orieux F, Féron O, Giovannelli JF. Sampling high-dimensional Gaussian distributions for general linear inverse problems. IEEE Signal Processing Letters. 2012;19(5):251-254.

[38] Gilavert C, Moussaoui S, Idier J. Efficient Gaussian sampling for solving large-scale inverse problems using MCMC. IEEE Transactions on Signal Processing. 2015;63(1):70-80.

[39] Pereyra M, Dobigeon N, Batatia H, Tourneret JY. Segmentation of skin lesions in 2-D and 3-D ultrasound images using a spatially coherent generalized Rayleigh mixture model. IEEE transactions on medical imaging. 2012;31(8):1509-1520.

[40] Gonzalez J, Low Y, Gretton A, Guestrin C. Parallel Gibbs Sampling: From Colored Fields to Thin Junction Trees. In: AISTATS. vol. 15; 2011. p. 324-332.

[41] Boykov Y, Veksler O, Zabih R. Fast approximate energy minimization via graph cuts. IEEE Transactions on pattern analysis and machine intelligence. 2001;23(11):1222-1239.

[42] Greig DM, Porteous BT, Seheult AH. Exact maximum a posteriori estimation for binary images. Journal of the Royal Statistical Society Series B (Methodological). 1989;p. 271-279.

[43] Ford LR, Fulkerson D. Flow in networks. Princeton University Press; 1962.

[44] Strandmark P, Kahl F. Parallel and distributed graph cuts by dual decomposition. In: Computer Vision and Pattern Recognition (CVPR), 2010 IEEE Conference on. IEEE; 2010. p. 2085-2092.

[45] Goldschlager LM, Shaw RA, Staples J. The maximum flow problem is log space complete for P. Theoretical Computer Science. 1982;21(1):105-111.

[46] Besag J. On the statistical analysis of dirty pictures. Journal of the Royal Statistical Society Series B (Methodological). 1986;p. 259-302.

[47] Held K, Kops ER, Krause BJ, Wells WM, Kikinis R, Muller-Gartner HW. Markov random field segmentation of brain MR images. IEEE transactions on medical imaging. 1997;16(6):878-886.

[48] Zhang Y, Brady M, Smith S. Segmentation of brain MR images through a hidden Markov random field model and the expectation-maximization algorithm. IEEE transactions on medical imaging. 2001;20(1):45-57. 
[49] Huang F, Narayan S, Wilson D, Johnson D, Zhang GQ. A Fast Iterated Conditional Modes Algorithm for Water-Fat Decomposition in MRI. IEEE transactions on medical imaging. 2011;30(8):1480-1492.

[50] Geman S, Geman D. Stochastic relaxation, Gibbs distributions, and the Bayesian restoration of images. IEEE Transactions on pattern analysis and machine intelligence. 1984;(6):721-741.

[51] Schmidt M, Alahari K. Generalized fast approximate energy minimization via graph cuts: Alpha-expansion beta-shrink moves. arXiv preprint arXiv:11085710. 2011;.

[52] Shepp LA, Logan BF. The Fourier reconstruction of a head section. IEEE Transactions on Nuclear Science. 1974;21(3):21-43.

[53] Gay L, Arslan P, Rambourg Y, Chandelle A, inventors; Fantôme destiné à être utilisé pour le contrôle de la qualité d'images tomographiques. 3030753; 2016.

[54] Gac N, Mancini S, Desvignes M, Houzet D. High speed 3D tomography on CPU, GPU, and FPGA. EURASIP Journal on Embedded systems. 2008;2008:5.

[55] Peters T. Algorithms for fast back-and re-projection in computed tomography. IEEE transactions on nuclear science. 1981;28(4):3641-3647.

[56] Joseph PM. An improved algorithm for reprojecting rays through pixel images. IEEE transactions on medical imaging. 1982;1(3):192-196.

[57] Siddon RL. Fast calculation of the exact radiological path for a three-dimensional CT array. Medical physics. 1985;12(2):252-255.

[58] Jacobs F, Sundermann E, De Sutter B, Christiaens M, Lemahieu I. A fast algorithm to calculate the exact radiological path through a pixel or voxel space. CIT Journal of computing and information technology. 2015;6(1):89-94.

[59] Zeng GL, Gullberg GT. Unmatched projector/backprojector pairs in an iterative reconstruction algorithm. IEEE transactions on medical imaging. 2000;19(5):548-555.

[60] MacQueen J. Some methods for classification and analysis of multivariate observations. In: Proceedings of the fifth Berkeley symposium on mathematical statistics and probability. vol. 1. Oakland, CA, USA.; 1967. p. 281-297.

[61] Kurugollu F, Sankur B, Harmanci AE. Color image segmentation using histogram multithresholding and fusion. Image and vision computing. 2001;19(13):915-928.

[62] Koontz WLG, Narendra PM, Fukunaga K. A graph-theoretic approach to nonparametric cluster analysis. IEEE Transactions on Computers. 1976;100(9):936-944.

[63] Pereyra M, Dobigeon N, Batatia H, Tourneret JY. Estimating the granularity coefficient of a Potts-Markov random field within a Markov chain Monte Carlo algorithm. Image Processing, IEEE Transactions on. 2013;22(6):2385-2397.

[64] Pereyra M, Whiteley N, Andrieu C, Tourneret JY. Maximum marginal likelihood estimation of the granularity coefficient of a Potts-Markov random field within an mcmc algorithm. In: 2014 IEEE Workshop on Statistical Signal Processing (SSP). IEEE; 2014. p. 121-124.

[65] Goldstein T, Osher S. The split Bregman method for L1-regularized problems. SIAM journal on imaging sciences. 2009;2(2):323-343.

[66] Bregman LM. The relaxation method of finding the common point of convex sets and its application to the solution of problems in convex programming. USSR computational mathematics and mathematical physics. 1967;7(3):200-217.

[67] Rosu RG, Giovannelli JF, Giremus A, Vacar C. Potts model parameter estimation in Bayesian segmentation of piecewise constant images. In: 2015 IEEE International Conference on Acoustics, Speech and Signal Processing (ICASSP). IEEE; 2015. p. 4080-4084. 